\title{
Spin accumulation and Andreev reflection in a mesoscopic ferromagnetic wire
}

\author{
W. Belzig \\ Department of Applied Physics and Delft Institute of Microelectronics and Submicrontechnology, Delft University of Technology, \\ Lorentzweg 1, 2628 CJ Delft, The Netherlands \\ Arne Brataas \\ Lyman Laboratory of Physics, Harvard University, Cambridge, Massachusetts 02138 \\ Yu. V. Nazarov and Gerrit E. W. Bauer \\ Department of Applied Physics and Delft Institute of Microelectronics and Submicrontechnology, Delft University of Technology, \\ Lorentzweg 1, 2628 CJ Delft, The Netherlands
}

(Received 7 May 2000)

\begin{abstract}
The electron transport though ferromagnetic-metal-superconducting hybrid devices is considered in the nonequilibrium Green's function formalism in the quasiclassical approximation. Attention is focused on the limit in which the exchange splitting in the ferromagnet is much larger than the superconducting energy gap. Transport properties are then governed by an interplay between spin accumulation close to the interface and Andreev reflection at the interface. We find that the resistance can be either enhanced or lowered in comparison to the normal case and can have a nonmonotonic temperature and voltage dependence. In the nonlinear voltage regime electron heating effects may govern the transport properties, leading to qualitative different behavior than in the absence of heating effects. Recent experimental results on the effect of the superconductor on the conductance of the ferromagnet can be understood by our results for the energy-dependent interface resistance together with effects of spin accumulation without invoking long-range pairing correlations in the ferromagnet.
\end{abstract}

\section{INTRODUCTION}

Much theoretical and experimental work has addressed the effect of a superconductor $(\mathrm{S})$ in proximity to a normal metal $(\mathrm{N})$ on the transport properties during the last years; see Ref. 1 and references therein for an overview. Most experimental results can be explained in the framework of the quasiclassical theory of superconductivity accounting for a "long-range" proximity effect with a coherence length $\xi$ $=\left(\hbar D / 2 k_{\mathrm{B}} T\right)^{1 / 2}$, where $D$ is the diffusion coefficient of the normal metal and $T$ is the temperature. On the other hand, applications of the quasiclassical theory to transport in heterostructures containing ferromagnets $(\mathrm{F})$ are still scarce. In contrast to normal metals the presence of a strong exchange field in the ferromagnet leads to a strong difference in the energy dispersions for the two spin bands. However, longrange coherence in normal metals requires spin-degenerate bands close to the Fermi energy, since singlet superconductivity couples quasiparticles of different spins by Andreev reflection. The consequence of the exchange field energy $h_{\mathrm{xc}}$ is a strong decoherence of quasiparticles belonging to the different spin bands. Typically the superconducting energy scale $\Delta$ is smaller than $h_{\mathrm{xc}}$ by several orders of magnitude for $(\mathrm{Al}, \mathrm{Nb}) \mathrm{vs}(\mathrm{Fe}, \mathrm{Ni}$, and $\mathrm{Co})$, respectively. Thus, the proximity effect in ferromagnetic metals is negligible and a ferromagnet in contact with a superconductor may be considered as an incoherent metal coupled to the superconductor. In this case all changes induced by the contact to a superconductor depend on the properties of the interface itself. This is accomplished by the effect of spin accumulation, ${ }^{2,3}$ which does not require phase coherence in the ferromagnet and can therefore have a much longer range than the proximity effect. The main purpose of this paper is to study the mutual influence of resistance changes by spin accumulation and interface properties.

Recently heterostructures of ferromagnets and superconductors have been experimentally realized and investigated. $^{4-7}$ Several unusual phenomena have been unveiled. The experimental results in point contact geometries ${ }^{4}$ can be explained by the reduced, bias-dependent transparency of the interface due to spin-dependent band mismatch between the normal metal and the ferromagnet. ${ }^{4,8}$ The experimental results in diffusive nanostructured samples ${ }^{5-7}$ are more intriguing. The measured conductance changes on the ferromagnetic side can be positive and negative at the superconducting transition with amplitudes much larger than anticipated. The sign and the amplitude of the changes appear to depend strongly on the ferromagnetic-superconductor interface transparency. It has been conjectured that a strong mutual influence of the superconductors and ferromagnetic conductors and a penetration of the superconducting order parameter into the ferromagnet over distances many times longer than expected from the above estimates might explain the observations. ${ }^{5-7}$

Some effects of the interplay between spin accumulation and Andreev reflection in diffuse systems have been discussed in Ref. 9. Since the spin current into a superconductor vanishes at sufficiently low bias and temperature, a nonequilibrium spin accumulation builds up on the ferromagnetic side in order to conserve the spin currents. The spin accumulation causes an additional boundary resistance which is of the order of the resistance of the ferromagnetic wire of a 
length of the spin-flip diffusion length. Therefore the resistance of the F-S system should be always larger than that of the the F-N system, in contradiction with some of the experimental observations. The reason for this apparent failure is the assumption that the resistance of the FS interface is negligible compared to other changes of the resistance. Previous theories took into account only perfectly ballistic interfaces for which the resistance is determined purely by the matching of the adjacent Fermi surfaces. The interface resistance and its modulation are then of the order of the Sharvin resistance, which is negligible compared to the total one. However, in sputtered samples with relatively large contact areas $^{5-7}$ the interface can contribute significantly, especially when differences of resistances below and above the superconducting transition temperatures are considered.

Other transport phenomena in ferromagnetsuperconductor systems have been studied in Refs. 10-12. In the first two references the influence of the proximity effect on the zero-temperature conductance was studied under the assumption that the ferromagnet has only a small exchange splitting (of the order of or smaller than the superconducting gap). In the ferromagnetic metals that we have in mind, this is not the case and we can therefore neglect the proximity effect. References 12 are generalizations of the well-known Blonder-Tinkham-Klapwijk (BTK) model ${ }^{13}$ of an interface potential to the case of a ferromagnet-(un)conventionalsuperconductor point contact. These results rely on atomically sharp interfaces without disorder, and cannot be used to describe the experiments ${ }^{5-7}$ that we have in mind.

It has been speculated that the triplet component of the order parameter induced by the fluctuations of the spin-orbit scattering potentials is essential in mesoscopic junctions. ${ }^{14}$ Neglecting magnetic impurities and spin-orbit coupling the superconducting order parameter is a spin singlet. However, magnetic impurities or spin-orbit coupling induce a fluctuating spin-triplet component with zero average. The triplet component is "long-range" coherent in the ferromagnet since it couples electrons and holes with the same spin and the exchange field in the ferromagnet does not play a role. However, the contribution to the conductivity from the triplet fluctuations is only relevant when the fluctuations are relatively large which is only the case when the conductance is close to the quantum conductance. The experimental samples ${ }^{5-7}$ have a much larger conductance, and we do not expect that such mesoscopic fluctuations play an important role.

None of the above-mentioned theories can explain the recent experimental results. This makes it necessary to study the properties of the contact between the ferromagnet and the normal metal in more detail and to account for a possible spin accumulation and heating effects in the ferromagnet including different interface morphologies. In particular we will go beyond the assumption of a perfect transparent metallic interface ${ }^{9}$ and discuss its influence on the observed conductance changes below the superconducting transition temperature and for bias voltages less than the superconducting gap. We will in this work radically disregard the proximity effect. Therefore our results only apply to ferromagnets with $h_{\mathrm{xc}} \gg \Delta$, which is, e.g., the case for the magnetic transition metals ( $\mathrm{Fe}, \mathrm{Co}$, and $\mathrm{Ni}$ ) in conjunction with superconducting metals like $\mathrm{Nb}$ and $\mathrm{Al}$. This assumption is supported

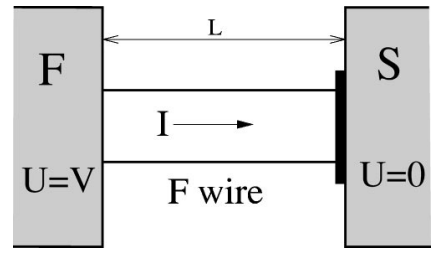

FIG. 1. Schematic layout of the mesoscopic ferromagnetic wire. It is placed between a ferromagnetic reservoir held at voltage $V$ and a grounded superconducting reservoir. The contact to the superconductor is an arbitrary connector, characterized by spin-dependent conductances in the normal state.

by the experimental fact that FS interferometers show no phase-periodic oscillations down to the level of $0.1 e^{2} / h$ in strong ferromagnets. ${ }^{5-7}$ In contrast to the calculations presented in this paper, the proximity effect could be important in weak ferromagnets. Below we will show that most of the recent experimental results can be explained in terms of the energy dependence introduced by the interface and the accompanying change in the spin accumulation. It is important to note that these changes are small in comparison to the total resistance, which is dominated by the long ferromagnetic wire. Nevertheless, they play a dominant role in the resistance modulation.

The paper is organized in the following way: Section II gives a description of the diffusive ferromagnetic wire both in the limit of elastic and inelastic scattering between the electrons. Section III treats the boundary condition between the ferromagnet and the superconductor which is crucial for the understanding of the transport properties. The results for the conductance obtained from the description of the ferromagnetic wire with these boundary conditions are discussed in Sec. IV. Finally we compare our results with experiments in Sec. V and give our conclusions in Sec. VI.

\section{DESCRIPTION OF THE FERROMAGNET}

We consider a ferromagnetic diffusive wire connected to an ideal (ferromagnetic or normal metal) reservoir on one side and to a superconducting reservoir on the other side as depicted in Fig. 1. The wire is characterized by length $L$, cross section $A$, and spin-dependent conductivities $\sigma_{\uparrow}$ and $\sigma_{\downarrow}$. In this section we discuss the kinetic equations describing the ferromagnetic wire in the absence of the proximity effect. We consider collisions with impurities to be the dominant scattering processes and use the diffusion approximation. The electrons in the quasi-one-dimensional wire are described by energy $\epsilon$ and spatial $x$ dependent distribution functions $f_{s}(\epsilon, x)$ for the two spin directions $s=+,-$ for spin $\uparrow$ and $\downarrow$, respectively. The distribution functions obey two coupled Boltzmann equations in the diffusive limit. Other scattering mechanisms will be specified in the following subsections.

Instead of the spin-dependent conductivities, it is convenient to introduce the total conductivity $\sigma=\sigma_{\uparrow}+\sigma_{\downarrow}$ and the spin polarization of the conductivity, $\gamma=\left(\sigma_{\uparrow}-\sigma_{\downarrow}\right) / \sigma$. The spin-dependent conductivities are then expressed as $\sigma_{s}$ 
$=(1+s \gamma) \sigma / 2$. We will also make use of the total conductance (resistance) of the ferromagnetic wire $G_{\mathrm{F}}$ $=A \sigma / L\left(R_{\mathrm{F}}=1 / G_{\mathrm{F}}\right)$.

\section{A. Elastic scattering}

In the elastic scattering case the energy is conserved in the scattering processes. This makes it necessary to study the energy-dependent distribution functions in the ferromagnetic wire. In addition to elastic impurity scattering we consider spin-flip scattering processes accounted for by the spin-flip length $l_{\text {sf }}$. Then, the kinetic equations read

$$
\frac{d^{2}}{d x^{2}} f_{s}(\epsilon, x)=\frac{1}{l_{\mathrm{sf}}^{2}}\left[f_{s}(\epsilon, x)-f_{-s}(\epsilon, x)\right] .
$$

The current for spin $s$ is given by $(e=|e|)$

$$
I_{s}(x)=-\sigma_{s} \frac{A}{e} \int d \epsilon \frac{d f_{s}(\epsilon, x)}{d x}=\int d \epsilon I_{s}(\epsilon, x) .
$$

This equation defines the spectral current $I_{s}(\epsilon, x)$. Electrical and spin currents are $I_{\text {charge }}=I_{\uparrow}(x)+I_{\downarrow}(x)$ and $I_{\text {spin }}(x)$ $=I_{\uparrow}(x)-I_{\downarrow}(x)$ and similar for the spectral currents. It is convenient to introduce the conductivity-averaged distribution function $\sigma f_{\mathrm{el}}(\epsilon, x)=\sigma_{\uparrow} f_{\uparrow}(\epsilon, x)+\sigma_{\downarrow} f_{\downarrow}(\epsilon, x)$ and the nonequilibrium spin distribution function $f_{\mathrm{sp}}(\epsilon, x)=f_{\uparrow}(\epsilon, x)$ $-f_{\downarrow}(\epsilon, x)$. The kinetic equations in terms of these functions decouple and have the form of spectral current conservation and spatial relaxation of the nonequilibrium spin distribution, respectively.

\section{B. Inelastic scattering}

The reason to investigate the role of inelastic scattering is the convenient fact that the ferromagnet is an incoherent metal with rather strong correlations. Both phonon and electron-electron scattering can mediate inelastic scattering. In general it is not obvious which should dominate and both should be treated on equal footing. In order to achieve insight into the physics it is useful to consider limiting cases as well.

In the limit of strong inelastic scattering we assume that the electron-electron interaction is stronger than the electronphonon relaxation. When a bias voltage is applied, the local electron temperature can therefore be different from the temperature in the reservoirs. This transport regime is relevant when the typical inelastic scattering length is smaller than the spin-diffusion length.

The electrons relax to a local equilibrium

$$
f_{s}(\epsilon, x)=f\left(\epsilon ; \mu_{s}(x), T_{\mathrm{el}}(x)\right),
$$

where $\mu_{s}(x)$ is the spin-dependent chemical potential, $T_{\mathrm{el}}(x)$ is the local temperature, and

$$
f(\epsilon ; \mu, T)=\frac{1}{1+\exp \left[(\epsilon-\mu) / k_{\mathrm{B}} T\right]}
$$

is the Fermi-Dirac distribution function. This makes it possible to integrate the kinetic equation and the currents over energy and to obtain equations for the local chemical potentials and electron temperature.
The spin-dependent (electric) current from Eq. (2) is

$$
I_{s}(x)=-\frac{\sigma}{2 e}(1+s \gamma) \frac{d \mu_{s}(x)}{d x} .
$$

Current conservation requires

$$
\frac{d^{2}}{d x^{2}}\left[\sigma_{\uparrow} \mu_{\uparrow}(x)+\sigma_{\downarrow} \mu_{\downarrow}(x)\right]=0 .
$$

Spin relaxation occurs within the spin-diffusion length $l_{\mathrm{sf}}$ :

$$
\frac{d^{2}}{d x^{2}}\left[\mu_{\uparrow}(x)-\mu_{\downarrow}(x)\right]=\frac{1}{l_{\mathrm{sf}}^{2}}\left[\mu_{\uparrow}(x)-\mu_{\downarrow}(x)\right] .
$$

The local spin-dependent chemical potentials in the ferromagnet are determined by Eqs. (6) and (7) and the boundary conditions to be discussed below.

Additionally, we need equations describing energy transport in the system to account for heating of the electrons. The energy current is

$$
\begin{aligned}
I_{\epsilon}(x) & =-\frac{A}{e^{2}} \sum_{s} \sigma_{s} \int d \epsilon \epsilon \frac{d f_{s}(\epsilon, x)}{d x} \\
& =\left[\mu_{\uparrow}(x) I_{\uparrow}(x)+\mu_{\downarrow}(x) I_{\downarrow}(x)\right] / e+I^{Q}(x),
\end{aligned}
$$

where the heat current is

$$
I_{\mathrm{Q}}(x)=-\kappa_{\mathrm{Q}}(x) A \frac{d T_{\mathrm{el}}(x)}{d x}
$$

the heat conductivity $\kappa_{\mathrm{Q}}(x)=\sigma \mathcal{L}_{0} T_{\mathrm{el}}(x)$, and the Lorentz number is $\mathcal{L}_{0}=\left(\pi^{2} / 3\right)\left(k_{\mathrm{B}} / e\right)^{2}$.

The conservation law for the energy dictates

$$
\frac{d}{d x} I_{\epsilon}(x)=A\left(\frac{\partial \rho_{\epsilon}(x)}{\partial t}\right)_{\text {rel }},
$$

where $\rho_{\epsilon}(x)$ is the local energy density. The energy relaxation between the electronic system and the phonons at sufficiently low temperatures is ${ }^{15}$

$$
\left(\frac{\partial \rho_{\epsilon}(x)}{\partial t}\right)_{\text {rel }}=\zeta\left\{\left(k_{\mathrm{B}} T\right)^{5}-\left[k_{\mathrm{B}} T_{\mathrm{el}}(x)\right]^{5}\right\},
$$

where $\zeta$ parametrizes the strength of the electron-phonon interaction, $\zeta=48 \pi \zeta(5) N\left(\epsilon_{F}\right) \lambda^{*} /\left(\hbar^{3} \omega_{D}^{2}\right), \quad \zeta(5) \approx 1.04$ is the Riemann zeta function, $N\left(\epsilon_{F}\right)$ is the density of states of both spins per unit cell, $\lambda *$ is of the same order of magnitude as the electron-phonon coupling constant $\lambda$, and $\hbar \omega_{D}$ is the Debye energy.

The conservation of energy, Eq. (10), together with the expression for the energy relaxation, Eq. (11), give a differential equation for the local electron temperature which can be solved together with the boundary conditions to be discussed below.

When the electron-phonon interaction is weak there is no exchange of energy between the electron and the phonon systems so that the right hand side of Eq. (10) can be set to zero and we have conservation of the energy current due to the electron transport $d I^{e}(x) / d x=0$. In the opposite limit of a strong electron-phonon interaction the electron temperature 
equals the lattice temperature. The differential equation for the energy conservation with the boundary conditions given above can in these two cases be solved exactly. In the intermediate regime the equations will be solved numerically.

\section{BOUNDARY CONDITIONS}

The condition that the ferromagnet should be completely incoherent leads to simplified boundary conditions for the kinetic equations. These boundary conditions can be derived from the boundary conditions for the quasiclassical Green's function. ${ }^{16}$ A transparent form suitable for diffusive systems has been presented by Nazarov. ${ }^{17}$ We will follow the spirit and the notation of this paper. A circuit theory for ferromagnetic-normal-metal systems has been presented in Ref. 18. A contact is described by a set of transmission eigenvalues $\left\{T_{n}\right\}$ or, equivalently, by a distribution of the transmission eigenvalues $\rho(T)$. The boundary condition at the contact is expressed through a conservation law for the matrix current in the Keldysh formulation. In the framework of superconductivity it is a $4 \times 4$ matrix comprising $2 \times 2$ Keldysh space and $2 \times 2$ particle-hole (Nambu) space. Boundary conditions for any type of contact can be presented by means of this conservation law for the matrix current. For a contact characterized by transmission eigenvalues $\left\{T_{n}\right\}$ the matrix current has been derived in Ref. 17 and takes the form

$$
\check{I}=-\frac{2 e}{\pi \hbar} \sum_{n} T_{n} \frac{\left(\check{G}_{\mathrm{F}} \check{G}_{\mathrm{S}}-\check{G}_{\mathrm{S}} \check{G}_{\mathrm{F}}\right)}{4+T_{n}\left(\check{G}_{\mathrm{F}} \breve{G}_{\mathrm{S}}+\check{G}_{\mathrm{S}} \breve{G}_{\mathrm{F}}-2\right)} .
$$

This matrix current has to be equated to the diffusive matrix current entering the contact from either side. The two sides of the contact are characterized by the Keldysh matrix Green's functions $\breve{G}_{\mathrm{S}}$ and $\breve{G}_{\mathrm{F}}$, which we will specify to be the superconducting reservoir and the ferromagnetic wire, respectively. The Keldysh-Nambu matrix Green's function of the superconductor in equilibrium is

$$
\check{G}_{\mathrm{S}}(\epsilon)=\left(\begin{array}{cc}
\hat{G}_{\mathrm{S}}^{\mathrm{R}}(\epsilon) & \hat{G}_{\mathrm{S}}^{\mathrm{K}}(\epsilon) \\
0 & \hat{G}_{\mathrm{S}}^{\mathrm{A}}(\epsilon)
\end{array}\right) .
$$

A similar structure holds for any matrix in Keldysh space. In local equilibrium the Keldysh $(1,2)$ component in Nambu space is $\hat{G}_{\mathrm{S}}^{\mathrm{K}}(\epsilon)=\left[\hat{G}_{\mathrm{S}}^{\mathrm{R}}(\epsilon)-\hat{G}_{\mathrm{S}}^{\mathrm{A}}(\epsilon)\right]\left[1-2 f^{\mathrm{S}}(\epsilon)\right]$, where $f^{\mathrm{S}}(\epsilon)=\left[1+\exp \left(\epsilon / k_{\mathrm{B}} T\right)\right]^{-1}$ is the quasiparticle distribution function in the superconductor and we have set the chemical potential in the superconductor to zero. $\hat{G}_{\mathrm{S}}^{\mathrm{R}}(\epsilon)$ and $\hat{G}_{\mathrm{S}}^{\mathrm{A}}(\epsilon)$ are retarded and advanced Nambu Green's functions determining the spectral properties of the superconductor. In the BCS case with a real order parameter they are given by

$$
\hat{G}_{\mathrm{S}}^{\mathrm{R}}(\epsilon)=-\left[\hat{G}_{\mathrm{S}}^{\mathrm{A}}(\epsilon)\right]^{*}=\frac{(\epsilon+i 0) \hat{\tau}_{3}-i \Delta \hat{\tau}_{1}}{\sqrt{(\epsilon+i 0)^{2}-\Delta^{2}}} .
$$

The diagonal component represents the normal retarded Green's function whereas the off-diagonal component is conventionally called the anomalous Green's function. On the ferromagnetic side we completely neglect the proximity effect leading to the spectral functions $\hat{G}_{\mathrm{F}}^{\mathrm{R}}=\hat{\tau}_{3}=-\hat{G}_{\mathrm{F}}^{\mathrm{A}}$. The absence of an anomalous component is a result of the absence of the proximity effect. The Keldysh component accounts for the spin-dependent nonequilibrium distribution:

$$
\hat{G}_{\mathrm{F}}^{\mathrm{K}}(\epsilon)=2\left(\begin{array}{cc}
1-2 f_{\uparrow}^{\mathrm{F}}(\epsilon) & 0 \\
0 & 1-2 f_{\downarrow}^{\mathrm{F}}(-\epsilon)
\end{array}\right),
$$

where $f_{\uparrow}^{\mathrm{F}}(\epsilon)$ and $f_{\downarrow}^{\mathrm{F}}(\epsilon)$ are the quasiparticle distribution functions close to the interface on the ferromagnetic side. The spectral electrical current is determined by the Keldysh component of the matrix current according to

$$
I_{\mathrm{el}}(\epsilon)=\frac{1}{4 e} \operatorname{Tr}\left[\hat{\tau}_{3} \hat{I}^{\mathrm{K}}(\epsilon)\right] .
$$

Equations (15) and (16) suggest a representation of the diagonal components of the Keldysh component of the matrix current in the form

$$
\hat{I}^{\mathrm{K}}(\epsilon)=\left(\begin{array}{cc}
I_{\uparrow}(\epsilon) & \cdots \\
\cdots & I_{\downarrow}(-\epsilon)
\end{array}\right) .
$$

Now we are in the position to calculate the spin-resolved currents through the contact. Performing the calculations along the lines of Ref. 17 we find the spectral spin-dependent current

$$
\begin{aligned}
I_{s}(\epsilon)= & \frac{G_{\mathrm{QP}}(\epsilon)}{2 e}\left[f^{S}(\epsilon)-f_{s}^{F}(\epsilon)\right] \\
& +\frac{G_{\mathrm{A}}(\epsilon)}{4 e}\left[1-f_{s}^{F}(\boldsymbol{\epsilon})-f_{-s}^{F}(-\boldsymbol{\epsilon})\right] .
\end{aligned}
$$

The quasiparticle conductance $G_{\mathrm{QP}}(\epsilon)$ and the Andreev conductance $G_{A}(\epsilon)$ are determined by the properties of the contact and the spectral properties of the two metals connected by the contact. The distribution of transmission eigenvalues can be incorporated in a single characteristic complex function

$$
Z(x)=\frac{e^{2}}{\pi \hbar} \sum_{n} \frac{T_{n}}{2+T_{n}(x-1)},
$$

where $x(\epsilon)=\operatorname{Tr}\left\{\hat{G}_{\mathrm{S}}^{\mathrm{R}}(\boldsymbol{\epsilon}), \hat{G}_{\mathrm{F}}^{\mathrm{R}}(\boldsymbol{\epsilon})\right\} / 4$. The conductances are

$$
\begin{gathered}
G_{\mathrm{QP}}(\epsilon)=\operatorname{Re} Z(x) \operatorname{Re} x+\frac{\operatorname{Im} Z(x)}{\operatorname{Im} x} \operatorname{Im}^{2} \sqrt{1-x^{2}}, \\
G_{\mathrm{A}}(\epsilon)=-\frac{\operatorname{Im} Z(x)}{\operatorname{Im} x}\left|1-x^{2}\right| .
\end{gathered}
$$

The contact is characterized by a transmission distribution, which leads to contact-specific energy dependences of the conductances. The normal-state conductance is $G_{\mathrm{BN}}$ $=\left(e^{2} / 2 \pi \hbar\right) \Sigma_{n} T_{n}$. For a ballistic model contact all transmission eigenvalues are equal to 1 for the propagating channels and 0 otherwise and $\Sigma_{n} T_{n}=N$, where $N$ is the number of propagating channels. The distribution function in the case of a dirty interface is ${ }^{19}$

$$
\rho(T)=\frac{\hbar}{e^{2}} G_{\mathrm{BN}} \frac{1}{T^{3 / 2} \sqrt{1-T}}
$$


TABLE I. Spectral conductances of different generic contacts defined by the transmission distributions in the second column. The characteristic function $Z$ of the contact was defined in Eq. (19). As an example we present electrical and spin conductance for a contact between a ferromagnetic metal and a BCS superconductor in the last two columns. For energies below the superconducting gap $\Delta$ the quasiparticle conductance $G_{Q P}$ vanishes for all contacts. Note that the energy argument of all quantities $\epsilon$ is understood to be the absolute value of the energy.

\begin{tabular}{|c|c|c|c|c|c|}
\hline Contact & $\rho(T) / G_{\mathrm{N}}$ & $Z(\epsilon) / G_{\mathrm{N}}$ & \multicolumn{3}{|c|}{ F-S contact } \\
\hline & & & $\frac{G_{\mathrm{A}}}{G_{\mathrm{N}}}(\epsilon<\Delta)$ & $\frac{G_{\mathrm{A}}+G_{\mathrm{QP}}}{G_{\mathrm{N}}}(\epsilon>\Delta)$ & $\frac{G_{\mathrm{QP}}}{G_{\mathrm{N}}}(\epsilon>\Delta)$ \\
\hline Tunnel & $T_{n} \ll 1$ & 1 & 0 & $\frac{\epsilon}{\bar{\xi}}$ & $\frac{\epsilon}{\bar{\xi}}$ \\
\hline Ballistic & $T_{n}=1$ & $\frac{2}{1+x}$ & 2 & $\frac{2 \epsilon}{\epsilon+\xi}$ & $\frac{2 \xi}{\epsilon+\xi}$ \\
\hline Diffusive & $\frac{\hbar \pi}{2 e^{2}} \frac{1}{T \sqrt{1-T}}$ & $\frac{\arccos (x)}{\sqrt{1-x^{2}}}$ & $\frac{\Delta}{2 \epsilon} \ln \left(\frac{\Delta+\epsilon}{\Delta-\epsilon}\right)$ & $\frac{\epsilon}{2 \Delta} \ln \left(\frac{\epsilon+\Delta}{\epsilon-\Delta}\right)$ & 1 \\
\hline Dirty interface & $\frac{\hbar}{e^{2}} \frac{1}{T^{3 / 2} \sqrt{1-T}}$ & $\sqrt{\frac{2}{1+x}}$ & $\frac{\Delta}{\sqrt{\xi(\xi+\Delta)}}$ & $\sqrt{\frac{\epsilon+\xi}{2 \xi}}$ & $\frac{\sqrt{2} \epsilon}{\sqrt{\xi(\epsilon+\xi)}}$ \\
\hline Abbreviations & & $x=\frac{1}{4} \operatorname{Tr}\left\{\hat{G}_{\mathrm{S}}^{\mathrm{R}}, \hat{G}_{\mathrm{F}}^{\mathrm{R}}\right\}$ & & $2-\Delta^{2} \mid$ & \\
\hline
\end{tabular}

and in the case of a diffusive contact the distribution is ${ }^{20}$

$$
\rho(T)=\frac{\hbar}{2 e^{2}} G_{\mathrm{BN}} \frac{1}{T \sqrt{1-T}} .
$$

Finally, for a tunnel conductance a perturbation expansion in terms of the small transmission eigenvalues can be performed. We list the characteristic function $Z(x)$ for a number of generic contacts in Table I: tunnel junction, ballistic contact, diffusive contact, and dirty interface. In the case of an incoherent metal on one side (i.e., $\hat{G}_{\mathrm{F}}^{\mathrm{R}}=\hat{\tau}_{3}$ ), the argument of the characteristic function reduces to $x=\operatorname{Tr} \hat{\tau}_{3} \hat{G}_{\mathrm{S}}^{\mathrm{R}} / 2$. The result in this case is demonstrated explicitly in Table I for a contact of a BCS superconductor with spectral functions (14). The energy dependence of these spectral conductances is depicted in Fig 2. Below the superconducting gap only the Andreev conductance is nonzero, gradually decreasing from the value of $2 G_{\mathrm{BN}}$ for the metallic junction to zero in the tunnel junction. Above the gap the Andreev conductance vanishes rather quickly $\sim 1 / \epsilon^{2}$. Also quasiparticle transport becomes possible and, thus, spin transport into the superconductor.

The properties of these contacts are demonstrated by the temperature dependence of the linear conductance following from

$$
G_{\mathrm{BS}}(T)=\int d \epsilon\left[G_{\mathrm{QP}}(\epsilon)+G_{\mathrm{A}}(\epsilon)\right]\left(-\frac{\partial f(\epsilon, 0, T)}{\partial \epsilon}\right) .
$$

This is the conductance that would be measured if the contact were placed between a normal reservoir and a superconducting reservoir. The temperature dependence of the contact conductance (24) is shown in Fig. 3. The dashed and dotted lines show the conductance of the diffusive contact and the dirty interface, respectively. The resistance of the diffusive contact shows the well-known reentrant behavior; i.e., it reaches the normal-state conductance at zero temperature. ${ }^{21}$ The resistance of the dirty interface after a small drop below the critical temperature is higher than the normal-state value and saturates at low temperature at $\sqrt{2} R_{\mathrm{BN}} \cdot{ }^{19}$ The result for the dirty interface resembles that of Ref. 22, where this behavior was found with a different method for the resistance of a thin double barrier between a normal metal and a superconductor.

Additionally, we introduce mixed contacts as a model for an inhomogeneous interface with distributed regions with low and high transparency. The relative admixture $q$ of a
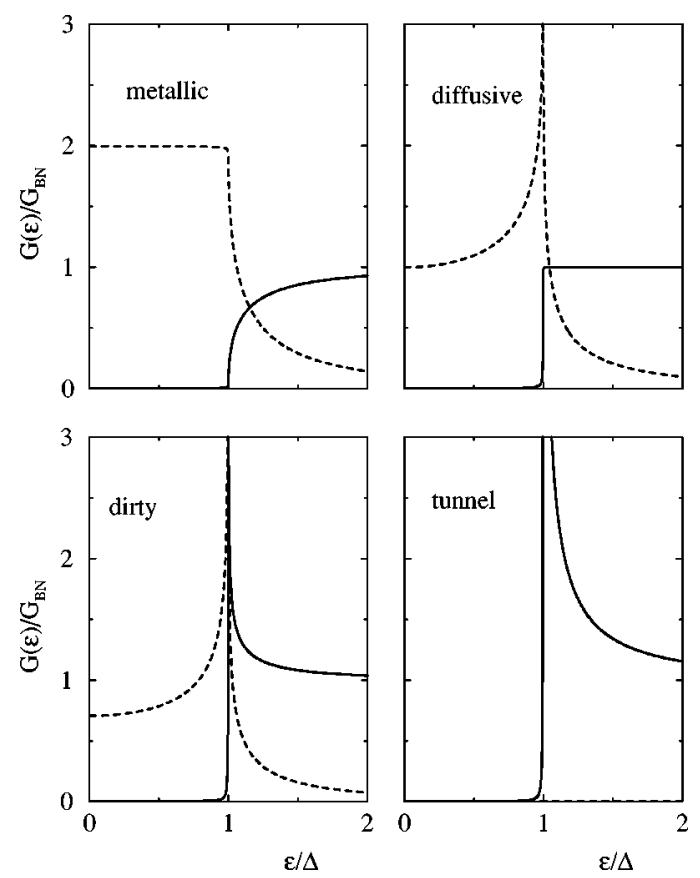

FIG. 2. Spectral conductances for different types of contacts. The solid curves denote the quasiparticle conductance $G_{\mathrm{QP}}(\epsilon)$ and the dashed curves the Andreev conductance $G_{\mathrm{A}}(\epsilon)$. The contact types as indicated in the figure are metallic junction (all $T_{n}=1$ ), diffusive contact [transmission distribution as defined in Eq. (23)], dirty interface [Eq. (22)], and tunnel junction (all $T_{n} \ll 1$ ). 


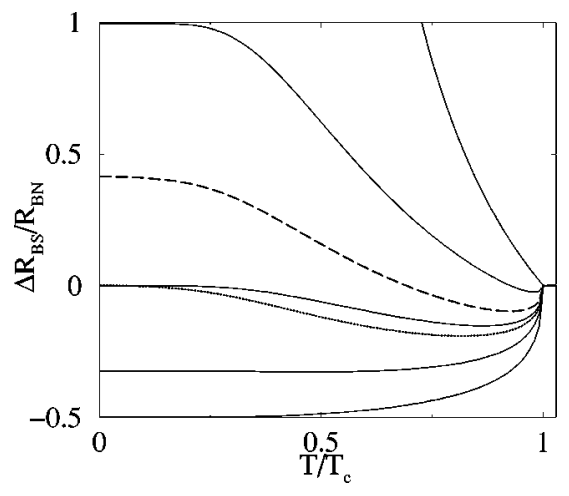

FIG. 3. Resistance change of different types of contacts between a normal metal reservoir and a superconducting reservoir. The mixed contact (solid line) varies from ballistic $(q=0)$ to tunnel $(q=1)$ from bottom to top. Intermediate values are $(q$ $=0.25,0.5,0.75)$. The diffusive contact is shown by the shortdashed line and the dirty contact by the long-dashed line (see Table I for a definition of these contacts).

tunnel and $(1-q)$ of a ballistic contact allows switching continuously from one limit to the other, covering approximately the universal cases of a diffusive contact $(q \approx 0.5)$ and a dirty interface $(q \approx 1 / \sqrt{2})$ with a single parameter $q$. Mathematically the contact is defined by

$$
G_{q}(\epsilon)=q G_{\text {tunnel }}(\epsilon)+(1-q) G_{\text {ballistic }}(\epsilon)
$$

for quasiparticle and Andreev conductance, respectively. The energy dependence of the individual conductances can be found in Table I. A common feature of the temperature dependence of all these contacts except the tunnel junction is that right below $T_{\mathrm{c}}$ the resistance drops. At lower temperatures the resistance increases again except in the case of the purely ballistic contact. The drop of resistance of these contacts close to $T_{\mathrm{c}}$ can be traced back to the temperature dependence of the superconducting order parameter $\Delta(T)$. The resistance drop is caused by the leading order contribution of the change in the superconducting gap $\Delta(T) \propto\left(1-T / T_{\mathrm{c}}\right)^{1 / 2}$ to the Andreev contribution and the conductance. ${ }^{23}$ A similar behavior is well known from Ref. 13, in which the transition from a ballistic to a tunneling contact is modeled by increasing a $\delta$-potential barrier at the interface.

The boundary conditions presented so far imply that the transmission ensembles and the number of channels are the same for the two spin species. In reality the transmission matrices for spin-up and spin-down states can be different. A microscopic calculation of the transmission eigenvalues is beyond the scope of the present paper. We will therefore heuristically generalize the boundary conditions to spindependent interfaces by taking different transmission ensembles for the two spin directions. These ensembles can differ in the total number of channels and/or in the transmission distribution. Thus, we replace the spin-dependent current through the interface (18) by

$$
\begin{aligned}
I_{\mathrm{s}}(\boldsymbol{\epsilon})= & \frac{G_{\mathrm{s}}(\boldsymbol{\epsilon})}{2 e}\left[f^{S}(\boldsymbol{\epsilon})-f_{\mathrm{s}}^{\mathrm{F}}(\boldsymbol{\epsilon}, 0)\right] \\
& +\frac{G_{\mathrm{A}}(\boldsymbol{\epsilon})}{4 e}\left[1-f_{\mathrm{S}}^{\mathrm{F}}(\boldsymbol{\epsilon}, 0)+f_{-\mathrm{s}}^{\mathrm{F}}(-\boldsymbol{\epsilon}, 0)\right] .
\end{aligned}
$$

In general the spin-dependent quasiparticle conductances $G_{\uparrow}(\epsilon)$ and $G_{\downarrow}(\epsilon)$ entering the first term are of different magnitude and have different energy dependences. Similarly as for the ferromagnetic wire we introduce the total conductance of the boundary $G_{B}(\epsilon)=G_{\uparrow}(\epsilon)+G_{\downarrow}(\epsilon)$ and a dimensionless factor $\gamma_{B}(\epsilon)=\left[G_{\uparrow}(\epsilon)-G_{\downarrow}(\epsilon)\right] / G_{B}(\epsilon)$, which we call polarization of the boundary conductance. Since the definitions (20) of quasiparticle and (21) of Andreev conductance have been derived for a spin-degenerate interface these definitions are not valid anymore for spin-dependent interface scattering. It is, however, reasonable to assume that the energy dependence of all conductances is well approximated by the same transmission ensemble, but different numbers of channels. We can motivate this choice by the fact that in the experiments that we have in mind the interfaces are strongly disordered regions, with a possible formation of an alloy layer extending over several monolayers. In such contacts the number of channels is more or less controlled by the differences of the cross sections of the Fermi surface. But, on the other hand, the transmission ensemble and, hence, the energy dependence of the conductance are not expected to vary much in typical disordered contacts on the scale of the superconductor gap.

We will in the following only take into account the differences in magnitude, but not in energy dependence. In the language of transmission distributions this means that the distributions are the same, but the number of channels differ. In this approximation the spin polarization of the boundary conductance $\gamma_{\mathrm{B}}$ is energy independent. The energy dependence of the Andreev conductance follows from the same transmission ensemble, but its magnitude will be reduced in comparison to the unpolarized case. It is important to notice that the boundary polarization and the polarization of the ferromagnetic wire need not have the same sign, since they are parametrically independent. The possibility of this is demonstrated by microscopic numerical calculations. ${ }^{24}$

\section{RESULTS AND DISCUSSIONS}

We solve the kinetic equations presented in Sec. II in the three cases: (A) purely elastic scattering, (B) inelastic scattering in linear response, and $(\mathrm{C})$ inelastic scattering in nonlinear response. The boundary condition on the superconducting side of the wire has been derived in Sec. III. The boundary conditions at the ferromagnetic reservoir are

$$
f_{\uparrow}(\epsilon,-L)=f_{\downarrow}(\epsilon,-L)=f(\epsilon ; e V, T),
$$

where $f(\epsilon ; e V, T)=\left\{\exp \left[(\epsilon-e V) / k_{\mathrm{B}} T\right]+1\right\}^{-1}$ is the FermiDirac equilibrium distribution at a constant voltage $V$ and temperature $T$.

In the case of inelastic scattering Eq. (27) also implies that the electron temperature equals the lattice temperature in the ferromagnetic reservoir $T_{\mathrm{el}}(x=-L)=T$. The other boundary condition for the electron temperature comes from the conservation of energy current in the ferromagnet and into the superconductor.

As a reference we calculate the resistance of the system in the normal state:

$$
R_{\mathrm{FN}}=R_{\mathrm{F}}+R_{\mathrm{BN}}+R_{\mathrm{sf}} \frac{\left(\gamma-\gamma_{\mathrm{B}}\right)^{2}}{1+R_{\mathrm{sf}} / R_{\mathrm{BN}}} .
$$


The third term is due to the spin accumulation in the ferromagnetic wire determined by the "spin-flip resistance" $R_{\mathrm{sf}}$ $=1 / G_{\mathrm{sf}}=R_{\mathrm{F}} l_{\mathrm{sf}} / L \tanh \left(l_{\mathrm{sf}} / L\right)\left(1-\gamma^{2}\right)$. In the limit of a weak ferromagnet $\gamma^{2} \ll 1$ and a short spin-flip relaxation length $l_{\mathrm{sf}} \leqslant L$ the spin-flip resistance reduces to $R_{\mathrm{sf}} \approx R_{\mathrm{F}} l_{\mathrm{sf}} / L$, i.e., the resistance of a piece of the ferromagnetic wire of length $l_{\text {sf }}$. We see that the excess resistance due to the spin accumulation increases with increasing asymmetry between the polarization of the bulk conductivity and the polarization of the interface conductance. The expression (28) will be used in the following to calculate resistance changes below the transition to the superconducting state:

$$
\Delta R_{\mathrm{FS}}(T, V)=R_{\mathrm{FS}}(T, V)-R_{\mathrm{FN}} .
$$

The differential resistance is defined by

$$
R_{\mathrm{FS}}(T, V)=\left(\frac{\partial I(T, V)}{\partial V}\right)^{-1}
$$

In the linear response regime we will omit the arguments of the differential resistance $R_{\mathrm{FS}} \equiv R_{\mathrm{FS}}(T, V \rightarrow 0)$.

In the following analysis it will be useful to define the following temperature-dependent average:

$$
\langle\cdots\rangle=\int_{-\infty}^{\infty} \ldots\left(-\frac{\partial f(\epsilon ; 0, T)}{\partial \epsilon}\right) d \epsilon .
$$

This average occurs, e.g., in the temperature-dependent conductances of a contact between an incoherent metal and a superconductor (24).

\section{A. Elastic scattering}

When the scattering in the wire is elastic, the general solution of Eq. (1) satisfying Eq. (27) may be written as

$$
\begin{aligned}
f_{\mathrm{el}}(\epsilon, x)= & \frac{G_{\mathrm{FS}}(\epsilon)}{G_{\mathrm{F}}}[f(\epsilon ; 0, T)-f(\epsilon ; e V, T)]\left(1+\frac{x}{L}\right) \\
& +f(\epsilon ; e V, T) .
\end{aligned}
$$

The spatially independent spectral conductance $G_{\mathrm{FS}}(\epsilon)$ determines the current through the structures and remains to be calculated. The solution of the kinetic equation (1) is found from the continuity of the spin currents into the superconductor (26) and ferromagnetic wire (2). We find the electrical current

$$
I(T, V)=\frac{1}{2 e} \int d \epsilon G_{\mathrm{FS}}(\epsilon)[1-f(\epsilon ; e V, T)-f(-\epsilon ; e V, T)] .
$$

This expression shows that the spectral conductance determines the transport in each energy slice depending on the difference in occupation of states at this energy in the reservoirs. This form is analogous to the classical definition of a conductance as the proportionality factor between current and voltage difference.

The spectral conductance is given by

$$
\begin{aligned}
\frac{1}{G_{\mathrm{FS}}(\epsilon)}= & \frac{1}{G_{\mathrm{F}}}+\frac{1}{G_{\mathrm{QP}}(\epsilon)+G_{\mathrm{A}}(\epsilon)} \\
& +\frac{\left(\gamma-\gamma_{\mathrm{B}} \frac{G_{\mathrm{QP}}(\epsilon)}{G_{\mathrm{QP}}(\epsilon)+G_{\mathrm{A}}(\epsilon)}\right)^{2}}{G_{\mathrm{sf}}+G_{\mathrm{QP}}(\epsilon)\left(1-\gamma_{\mathrm{B}}^{2} \frac{G_{\mathrm{QP}}(\epsilon)}{G_{\mathrm{QP}}(\epsilon)+G_{\mathrm{A}}(\epsilon)}\right)} .
\end{aligned}
$$

In the general case the full expression has to be used to calculate the resistance change in the superconducting state.

When the ferromagnetic wire dominates the resistance of the whole structure a simplified expression for the linear resistance change may be obtained. We first limit the discussion to the case of a weak ferromagnet and vanishing boundary polarization to obtain

$$
\begin{aligned}
\Delta R_{\mathrm{FS}}= & \left\langle\frac{1}{G_{\mathrm{QP}}(\epsilon)+G_{\mathrm{A}}(\epsilon)}\right\rangle-R_{\mathrm{BN}} \\
& +\gamma^{2}\left[\left\langle\frac{1}{G_{\mathrm{sf}}+G_{\mathrm{QP}}(\epsilon)}\right\rangle-\frac{1}{G_{\mathrm{sf}}+G_{\mathrm{BN}}}\right] .
\end{aligned}
$$

We see that the resistance change consists of two contributions. The first is the resistance change due to the change of the boundary resistance, which would also be present in the absence of spin polarization. Note, however, that this term can be qualitatively different from the case of a normal metal wire in contact with a superconductor since in this case the proximity effect would not be negligible. The second term accounts for the difference in spin accumulation between normal and superconducting state.

First we discuss the influence of spin accumulation on the FS resistance for a spin-degenerate interface. In Fig. 4 resistance changes for two types of contacts are shown for different polarizations of the ferromagnet. Solid curves are for a relatively good contact $(q=0.75)$ and dashed curves for a less transparent contact $(q=0.25)$. In this plot the total resistance of the system is dominated by the resistance of the ferromagnetic wire $R_{\mathrm{F}}=100 R_{\mathrm{BN}}$ and the spin-relaxation length is $l_{\mathrm{sf}}=0.03 \mathrm{~L}$, resulting in a spin-accumulation resistance $R_{\mathrm{sf}} \approx 3 R_{\mathrm{BN}}$. Accordingly, the resistance change is normalized to $R_{\mathrm{BN}}$ to show the relevant scale of the effect produced by the superconducting transition. For both contacts spin accumulation (increasing from the bottom to the top curves) leads to an enhancement of the resistance. Specifically the low-temperature resistance is well accounted for by Eq. (35) in the limit $T \rightarrow 0$ :

$$
\Delta R_{\mathrm{FS}}(T=0)=\frac{1}{G_{\mathrm{A}}(0)}-R_{\mathrm{BN}}+\gamma^{2} \frac{R_{\mathrm{sf}}}{1+R_{\mathrm{BN}} / R_{\mathrm{sf}}} .
$$

The third term of this equation shows that the spin accumulation always enhances the resistance, maximally by an amount $\gamma^{2} R_{\mathrm{sf}}$. The enhancement for the $q=0.25$ contact has a uniform temperature dependence and does not change qualitatively. This is different for the $q=0.75$ contact. Here the resistance decreases monotonically in the unpolarized case as a result of the Andreev-enhanced conductance. A small polarization $\gamma \approx 0.2-0.4$ results in a nonmonotonic temperature dependence, i.e., an increase of resistance at 


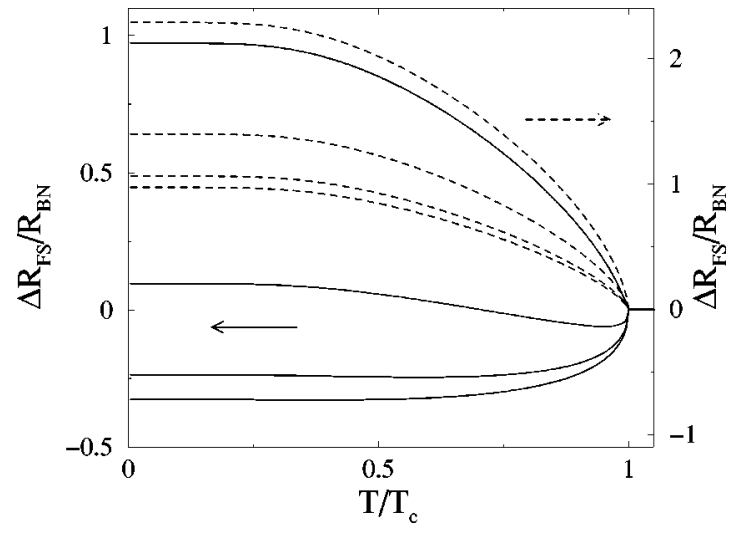

FIG. 4. Temperature dependence of the resistance change of the $\mathrm{F}$ wire attached to a superconducting reservoir. Results are presented for two mixed contacts with $q=0.75$ (solid lines) and $q$ $=0.25$ (dashed lines). The conductivity polarization $\gamma$ of the wire is changed from 0 to 0.6 in steps of 0.2 from the bottommost curve to the topmost curve for both contacts. Other parameters are $l_{\mathrm{sf}}$ $=0.03 L$, and $R_{\mathrm{F}}=100 R_{\mathrm{BN}}$. Clearly spin accumulation leads to an enhanced resistance in both cases. The resistance of the $q=0.25$ contact is more or less uniformly increased. The effect of spin accumulation is much more dramatic for the $q=0.75$ contact. The monotonic resistance decrease in the unpolarized case is first turned into an reentrant behavior for small polarization overshooting the normal-state resistance slightly at low temperatures. Increasing $\gamma$ further leads to an increased resistance for all temperatures. Note that this behavior resembles that of a $q=0.25$ contact, if properly rescaled.

lower temperatures. This can lead (for specific parameters) to a reentrant behavior of the resistance change, even overshooting the normal-state value for larger spin accumulation. At even higher spin polarizations $\gamma^{2} \gtrsim R_{\mathrm{BN}} / R_{\mathrm{sf}}$ the Andreev contribution is completely masked and the resistance increases monotonically. This behavior resembles that of a less transparent contact if the absolute scale is properly chosen.

Let us now discuss the effect of the interface polarization $\gamma_{\mathrm{B}}$ on the resistance change. In Fig. 5 the temperaturedependent resistance of a $q=0.75$ contact is shown for different interface polarizations. Other parameters are $R_{\mathrm{F}}$ $=100 R_{\mathrm{BN}}, l_{\mathrm{sf}}=0.03 L$, and $\gamma=0.3$. The interface polarization $\gamma_{\mathrm{B}}$ changes from the symmetric value +0.5 to the antisymmetric value -0.5 , as indicated in the plot. The reduction of the Andreev conductance by the spin-dependent interface resistance is taken into account by a phenomenological renormalization factor $\left(1-\gamma_{\mathrm{B}}^{2}\right)$. To gain some insight it is useful to look at the low-temperature limit of the resistance change in the limit $R_{\mathrm{BN}} \ll R_{\mathrm{sf}}$. From Eq. (34) it follows that

$$
\Delta R_{\mathrm{FS}}(T=0)=\frac{1}{G_{\mathrm{A}}(0)}-R_{\mathrm{BN}}+R_{\mathrm{sf}}\left(4 \gamma \gamma_{\mathrm{B}}-\gamma_{\mathrm{B}}^{2}\right)
$$

The spin-dependent contribution depends on the relative sign of the two polarizations $\gamma$ and $\gamma_{\mathrm{B}}$ and can also be negative (if $4 \gamma \gamma_{\mathrm{B}}<\gamma_{\mathrm{B}}^{2}$ ). This effect is seen in the lower two curves in Fig. 5 with an antisymmetric interface polarization. An increasing interface polarization leads to a lowering of the resistance change, despite the increase of the resistance due to the renormalization of the Andreev conductance. It is worth-

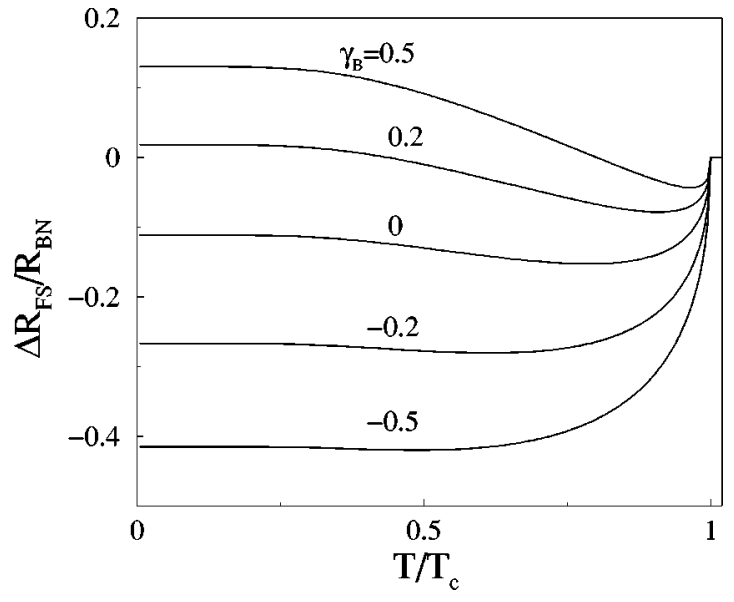

FIG. 5. Effect of the relative polarizations on the resistance change. The contact is a mixed contact with $q=0.75$; other parameters are $\gamma=0.3, l_{\mathrm{sf}}=0.03 L$, and $R_{\mathrm{F}}=100 R_{\mathrm{BN}}$. The polarization of the boundary conductance $\gamma_{\mathrm{B}}$ is varied between a symmetric configuration $\gamma_{\mathrm{B}}=0.5$ and an antisymmetric configuration $\gamma_{\mathrm{B}}$ $=-0.5$. At the same time the Andreev conductance is rescaled by a factor $1-\gamma_{B}^{2}$ to account for the smaller number and transmission of Andreev channels. For large antisymmetric polarization the resistance decrease exceeds the decrease of the corresponding normalmetal-superconductor contact.

while noting that for the largest negative interface polarization shown $\left(\gamma_{\mathrm{B}}=-0.5\right)$ the total resistance drop is larger than the resistance drop which would result from the pure Andreev reflection in the absence of spin polarization of the interface and the $\mathrm{F}$ wire. This apparent contradiction to the intuition that any spin accumulation should decrease the Andreev-caused resistance drop stems from the fact that we plot the resistance change below the superconducting transition. The contradiction is resolved by noting that the total resistance $R_{\mathrm{FS}}(T)=R_{\mathrm{FN}}+\Delta R_{\mathrm{FS}}(T)$ is always higher than for the unpolarized case. However, in a real experiment (with fixed polarizations) the Andreev conductance in the absence of a polarization cannot be measured separately. It may therefore appear that the measured resistance drop is larger than one would expect from a simple estimate of the reduction of the interface resistance due to Andreev reflection.

\section{B. Inelastic scattering-linear response}

We will now proceed to study the case of inelastic scattering in the ferromagnetic wire. It is assumed that the current in the ferromagnet is weakly polarized, $\gamma \ll 1$. In order to simplify the discussions we disregard the possible asymmetry in the interface transparency in the following discussions and set $\gamma_{\mathrm{B}}=0$. An extension is straightforward.

An analytical expression for the total conductance of the system can be found in the linear response regime. In this regime the effects of electron heating vanish since they will only contribute to the current in higher orders of the sourcedrain bias. The coupled equations for the spin-dependent chemical potential distributions and the electron temperature are simplified by letting $T_{\mathrm{el}}(x) \rightarrow T$. By solving Eqs. (6) and (7) together with the boundary conditions (18) and (27) we find the linear response resistance. Assuming a weak ferromagnet, $\gamma^{2} \ll 1$, and a small interface resistance compared to 
the resistance of the ferromagnetic wire $R_{\mathrm{BN}} \ll R_{\mathrm{F}}$ the resistance change can be written as

$$
\begin{aligned}
\Delta R_{\mathrm{FS}}(T)= & \frac{1}{\left\langle G_{\mathrm{QP}}(\epsilon)+G_{\mathrm{A}}(\epsilon)\right\rangle}-R_{\mathrm{BN}} \\
& +\gamma^{2}\left[\frac{1}{G_{\mathrm{sf}}+\left\langle G_{\mathrm{QP}}(\epsilon)\right\rangle}-\frac{1}{G_{\mathrm{sf}}+G_{\mathrm{BN}}}\right] .
\end{aligned}
$$

The first two terms in Eq. (38) are due to the effective interface resistance between the ferromagnet and the superconductor and the third term is due to the spin accumulation. The latter term vanishes when $\gamma \rightarrow 0$ or $l_{\mathrm{sf}} / L \rightarrow 0$. This equation has to compared with Eq. (29) for the case of purely elastic scattering. Only the quasiparticle conductance enters the spin-accumulation contribution since spins cannot be injected into the superconductor by means of the Andreev process. The temperature-averaged conductances directly determine the temperature dependence of the total resistance in the case of dominant inelastic scattering processes. The quasiparticle conductance vanishes at zero temperature since then no spin current can propagate into the superconductor. At zero temperature $\left\langle G_{\mathrm{QP}}(\epsilon)+G_{\mathrm{A}}(\epsilon)\right\rangle=G_{\mathrm{A}}(0)$ and the resistance of the FS system in the case of inelastic scattering, Eq. (38), equals the result in the case of elastic scattering, Eq. (36). If the first two terms in Eq. (38) are neglected and the contact is assumed to be ballistic, the results of Ref. 9 are reproduced.

The results with inelastic scattering in general differ from those with purely elastic scattering when the temperature is nonzero or when the current is measured in the nonlinear source-drain response regime. The remarkable difference between Eq. (29) and Eq. (38) is the way the thermal averaging is carried out. E.g., in the first term we have to average the inverse contact conductance in the case of elastic scattering, whereas we first have to average the conductance and then invert the result in the case of inelastic scattering. A similar consideration holds for the spin-accumulation term. The origin of this difference can be understood in the following way: we may visualize our wire (or any system) as mapped onto an electric circuit which contains energy-dependent conductors. In the case of purely elastic scattering we first have to calculate the total conductance of the system for each energy. The current is then found by averaging this spectral conductance with the difference of distribution functions of the adjacent reservoirs. This procedure yields Eq. (29) for the change in the resistance. In contrast, inelastic scattering equilibrates the local distribution of electrons in a way that the chemical potential is equal to the potential found from solving the circuit problem of the corresponding electric circuit. Thus, Eq. (38) follows Kirchhoff's laws for our system. As we will demonstrate below this difference can have significant consequences for the temperature dependence of the resistance.

Let us now illustrate the temperature dependence of the linear response conductance in the case of a metallic contact with an interface conductance much larger than the conductance of the ferromagnetic wire. The total conductance at sufficiently low temperatures is then $\left\langle G_{\mathrm{QP}}(\epsilon)+G_{\mathrm{A}}(\epsilon)\right\rangle$ $\approx G_{\mathrm{A}}(0)=2 G_{\mathrm{BN}}$ and the quasiparticle conductance is $\left\langle G_{\mathrm{QP}}(\epsilon)\right\rangle \approx\left(8 \pi k_{\mathrm{B}} T / \Delta\right)^{1 / 2} \exp \left(-\Delta / k_{\mathrm{B}} T\right)$. The temperature must then be so low that

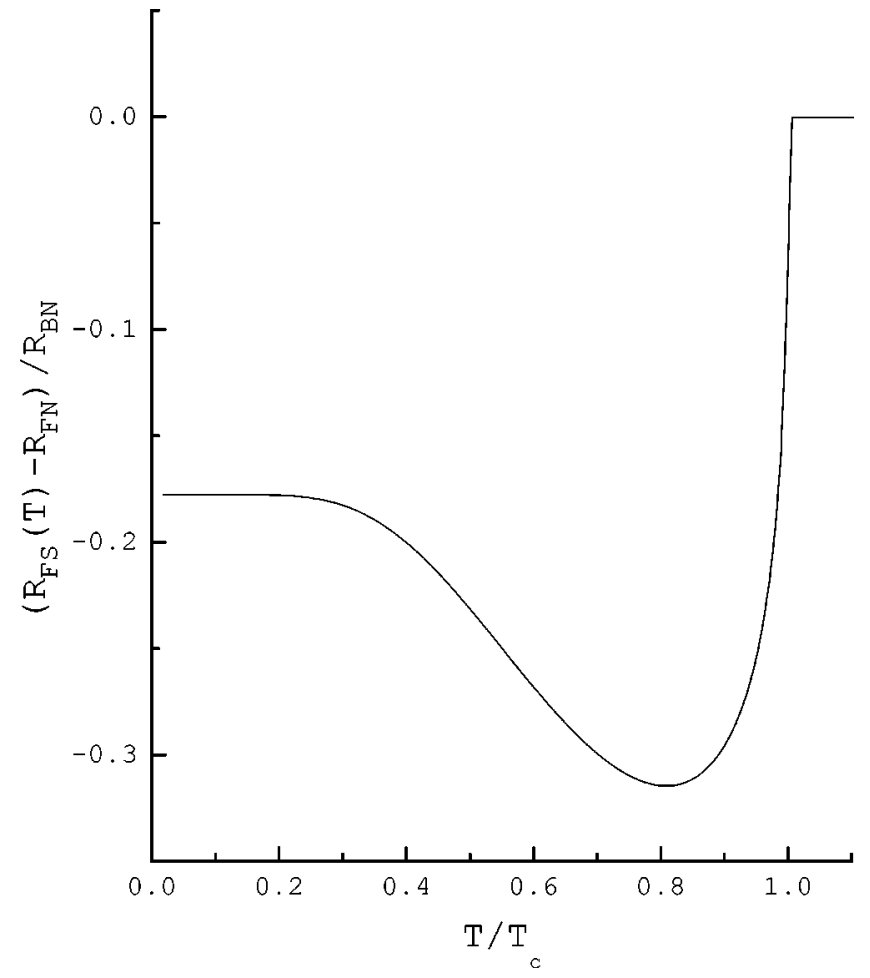

FIG. 6. The ratio of the resistance $R_{\mathrm{FS}}(T)$ to the interface resistance $R_{\mathrm{BN}}$ as a function of the reservoir temperature $T$. The ferromagnetic wire is parametrized by the polarization $\gamma=0.3$, the spinflip diffusion length $l_{\mathrm{sf}}=0.2 L$, and the interface resistance is $R_{\mathrm{BN}}$ $=0.05 R_{\mathrm{F}}$. The spin-flip resistance is $R_{\mathrm{sf}}=0.2 R_{\mathrm{F}}$.

$$
k_{\mathrm{B}} T \lesssim \frac{\Delta}{\ln \left(R_{\mathrm{BN}} / R_{\mathrm{sf}}\right)}
$$

in order to prevent a thermally assisted spin current into the superconductor.

We show in Fig. 6 the ratio of the linear response resistance change $\Delta R_{\mathrm{FS}}$ to the interface resistance $R_{\mathrm{BN}}$ as a function of the temperature $T$ for a metallic interface with $R_{\mathrm{BN}}$ $=0.05 R_{\mathrm{F}}$, polarization $\gamma=0.3$, and spin-flip diffusion length $l_{\mathrm{sf}} / L=0.2$. For these parameters we have a "spin-flip" resistance corresponding to $R_{\mathrm{sf}}=0.2 R_{\mathrm{F}}$. The change in resistance below the superconducting transition temperature is due to a competition between the excess resistance caused by the spin-flip relaxation and the reduced interface resistance caused by Andreev reflection. At $T=0$ we find from the approximate result (38) that $R_{\mathrm{FS}}-R_{\mathrm{FN}}=0.5 R_{\mathrm{BN}}-\gamma^{2} R_{\mathrm{sf}}$ $=-0.14 R_{\mathrm{FN}}$, roughly corresponding to the numerical value which has been obtained without making the approximation $\gamma^{2} \ll 1$ and $R_{\mathrm{F}} \gg R_{\mathrm{BN}}$. Using the condition (39) we find that the spin accumulation is strongly reduced around $T / T_{\mathrm{c}}=0.7$ and consequently the resistance of the system decreases before increasing again around $T / T_{\mathrm{c}}=1$ where the boundary resistance is increased. This explains the nonmonotonic behavior of the linear response resistance as a function of the temperature.

\section{Inelastic scattering-nonlinear response}

At a finite bias voltage the electron heating effects have to be taken into account and the coupled equations for the electron temperature, the spin-dependent chemical potentials (6), 


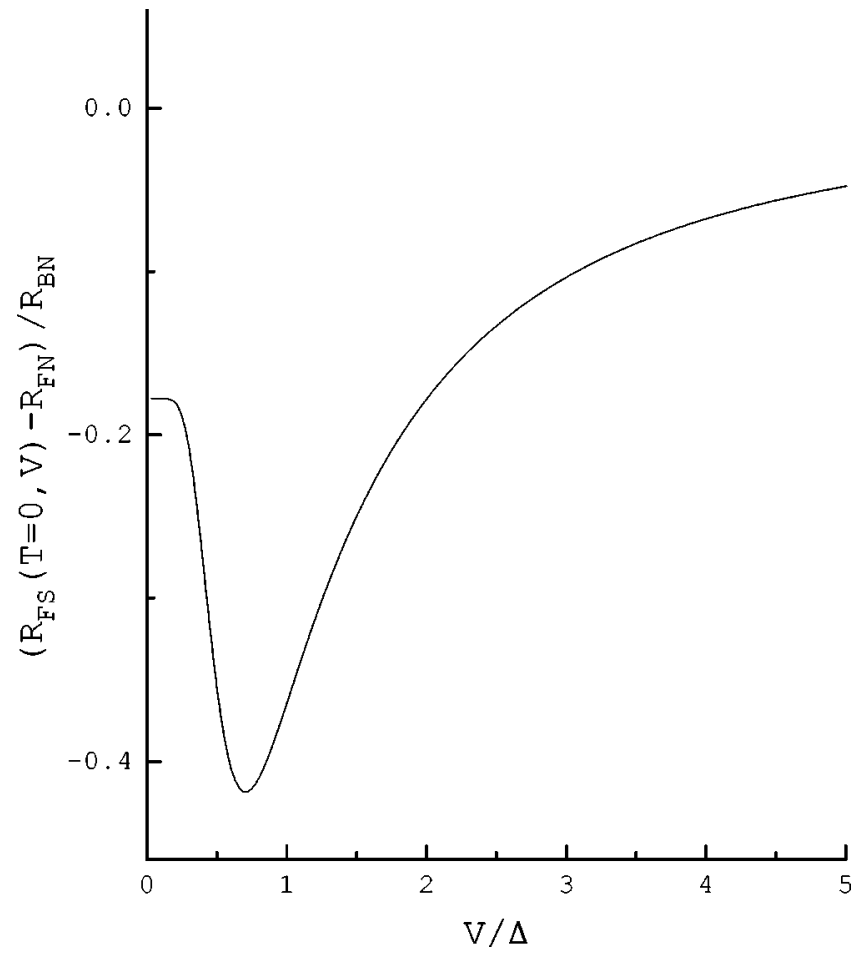

FIG. 7. The ratio of the resistance $R_{\mathrm{FS}}(T=0, V)$ to the interface resistance $R_{\mathrm{BN}}$ as a function of the bias voltage $V$. The ferromagnetic wire is parametrized by the polarization $\gamma=0.3$, the spin-flip diffusion length $l_{\mathrm{sf}}=0.2 L$, and the interface resistance is $R_{\mathrm{BN}}$ $=0.05 R_{\mathrm{F}}$. The spin-flip resistance is $R_{\mathrm{sf}}=0.2 R_{\mathrm{F}}$.

(7), (8), and (10), and the boundary conditions (18) and (27) have to be solved numerically.

First let us discuss the transport properties when the electron-phonon interaction is weak so that we have perfect conservation of energy current and the left hand side of Eq. (10) can be set to zero. From the discussions in the previous section we understand that there will be a reduction in the excess resistance due to the spin accumulation when the electron temperature on the ferromagnetic side reaches condition (39) so that there is a significant spin current entering the superconductor. Roughly speaking, the electron temperature on the ferromagnetic side is proportional to the applied source drain bias. Thus, as a crude approximation, we expect that the excess resistance due to the spin accumulation is lowered when

$$
e V \lesssim \frac{\Delta}{\ln \left(R_{\mathrm{BN}} / R_{\mathrm{sf}}\right)} .
$$

We show in Fig. 7 the resistance change $R_{\mathrm{FS}}(T=0, V)$ $-R_{\mathrm{BN}}$, Eq. (29), normalized by the interface resistance $R_{\mathrm{BN}}$ as a function of the bias voltage $V$. As before, the interface resistance is $R_{\mathrm{BN}}=0.05 R_{\mathrm{F}}$, the polarization $\gamma=0.3$, and the spin-flip diffusion length $l_{\mathrm{sf}} / L=0.2$. For these parameters we have a "spin-flip" resistance corresponding to $R_{\text {sf }}$ $=0.2 R_{\mathrm{F}}$. The change in resistance below the superconducting gap is due to a competition between the excess resistance caused by the spin-flip relaxation and the reduced interface resistance caused by the Andreev reflection. A dip in the resistance is seen around $V=0.7 \Delta$ which is correctly described by Eq. (40). Below this bias voltage the resistance is caused by the competition between spin accumulation which enhances the resistance and the effective interface resistance which reduces the resistance. At higher voltages the resistance is only caused by the effective interface resistance and the reduction of the resistance change $R_{\mathrm{FS}}(T=0, V)-R_{\mathrm{BN}}$ as a function of the bias voltage is small.

In the limit of a strong electron-phonon interaction the electron temperature equals the lattice temperature. The spin current into the superconductor is then not enhanced due to thermal activation and consequently the spin accumulation on the ferromagnetic side is only reduced when the potential on the ferromagnetic side of the interface is higher than the superconducting gap. This occurs when

$$
e V=\Delta\left(1+2 \frac{R_{\mathrm{F}}}{R_{\mathrm{BN}}}\right)
$$

and thus at a potential that is much larger than the superconducting gap, in contrast to the case of a weak electronphonon interaction.

In the intermediate regime the electron-phonon interaction should be included. In order to illustrate the main physics we consider the case of a weak polarization and set $\gamma=0$ and consequently there are no effects due to spin accumulation and the resistance change of the wire is only due to the change of the effective boundary resistance. The chemical potential in the ferromagnetic wire is thus spin independent. Furthermore, we consider the case that the lattice temperature is zero, $T=0$, so that the electron temperature arises solely due to electron heating. Solving the diffusion equation (6) on the ferromagnetic side of the interface gives $\mu(x)$ $=-e V x / L+\mu(0)[1+x / L]$, where $e V$ is the applied bias and $\mu(0)$ is the potential drop across the ferromagnetsuperconductor interface. The superconducting energy gap $\Delta$ presents a natural energy scale for the problem. We will characterize the strength of the electron-phonon energy exchange by a dimensionless constant $\kappa=A L \zeta e^{2} \Delta^{3} / G_{\mathrm{F}} ; \zeta$ is defined by the relation (11). The energy diffusion equation then simplifies to

$$
\begin{aligned}
\frac{\pi^{2}}{6}\left(L_{T} \partial_{x}\right)^{2}\left(k_{\mathrm{B}} T_{\mathrm{el}} / \Delta\right)^{2}= & \left(k_{\mathrm{B}} T_{\mathrm{el}} / \Delta\right)^{5} \\
& -\{[e V-\mu(0)] / \Delta\}^{2} / \kappa,
\end{aligned}
$$

where we introduce a typical length scale for the energy exchange $L_{T}=L / \sqrt{\kappa}$. If $\kappa \ll 1, L \ll L_{T}$ and the exchange is not effective. For longer wires, $\kappa$ becomes larger than unity. In this case, the electron temperature develops a constant plateau in the ferromagnetic wire and only changes rapidly within the length scale $L_{T}$ near the end points $x=-L$ and $x=0$. It follows from Eq. (42) that in this case the temperature in the middle of the ferromagnetic wire becomes

$$
\frac{k_{\mathrm{B}} T_{\mathrm{el}}}{\Delta}=\kappa^{-1 / 5}\left(\frac{e V-\mu(0)}{\Delta}\right)^{2 / 5} .
$$

We will now present numerical results of the temperature profile in the ferromagnetic wire and the resulting resistance change using $\gamma=0$, a metallic interface $R_{\mathrm{BN}} / R_{\mathrm{F}}=0.05$ for various values of the electron-phonon coupling constant. We show in Fig. 8 the spatially dependent electron temperature 


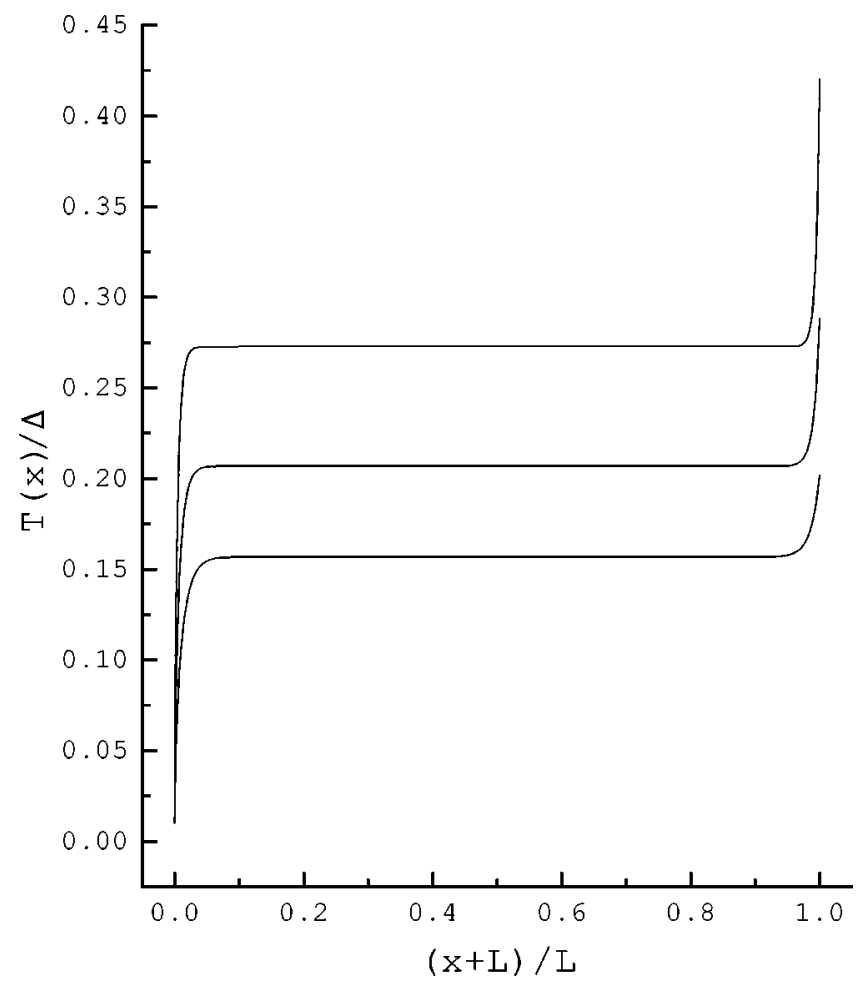

FIG. 8. The spatially dependent electron temperature in the ferromagnetic wire. The upper curve is for a bias voltage $V=40 \Delta$, the middle curve for a bias voltage $V=20 \Delta$, and the lower curve for a bias voltage $V=10 \Delta$. The metallic interface resistance is $R_{\mathrm{BN}}$ $=0.05 R_{\mathrm{F}}$, the reservoir temperature $T=0$, and the electron-phonon interaction strength $\kappa=10^{6}$.

in the ferromagnetic wire for $\kappa=10^{6}$ at a bias voltage $e V$ $=40 \Delta$ (upper curve), $e V=20 \Delta$ (middle curve), and $e V$ $=10 \Delta$ (lower curve). The electron temperature in the middle of the wire follows from Eq. (43). There are rapid changes of the electron temperature close to the ferromagnetic and superconducting reservoirs and the temperature in the middle of the wire is lower than the electron temperature close to the superconductor. The latter temperature is important for the effective interface resistance.

We show in Fig. 9 the resistance change as a function of the bias voltage. The different solid lines show the current for different ratios of the electron-phonon coupling starting from no electron-phonon interaction (a) $\kappa=0$ going through intermediate electron-phonon interaction (b) $\kappa=10^{2}$, (c) $\kappa$ $=10^{6}$, and (d) $\kappa=10^{8}$ to strong electron-phonon interaction (f) $\kappa=\infty$ when the electron temperature equals the lattice temperature, e.g., when there is no energy transfer between the electron and phonon system. The crossover bias voltage for the excess resistance is sensitive to the strength of the electron-phonon interaction and occurs from around $\Delta$ (a) to around $40 \Delta$ (f) [according to Eq. (41)]. The dependence on the electron-phonon interaction parameter $\kappa$ is rather weak as can be understood from Eq. (43). The local electron temperature in the middle of the ferromagnetic wire is proportional to $\kappa^{-1 / 5}$ and thus only has a very weak dependence on $\kappa$.

\section{DISCUSSION OF EXPERIMENTS}

In this section we discuss the connection of our results with experiments of Petrashov et al. ${ }^{5}$ and Giroud et al. ${ }^{6}$ It

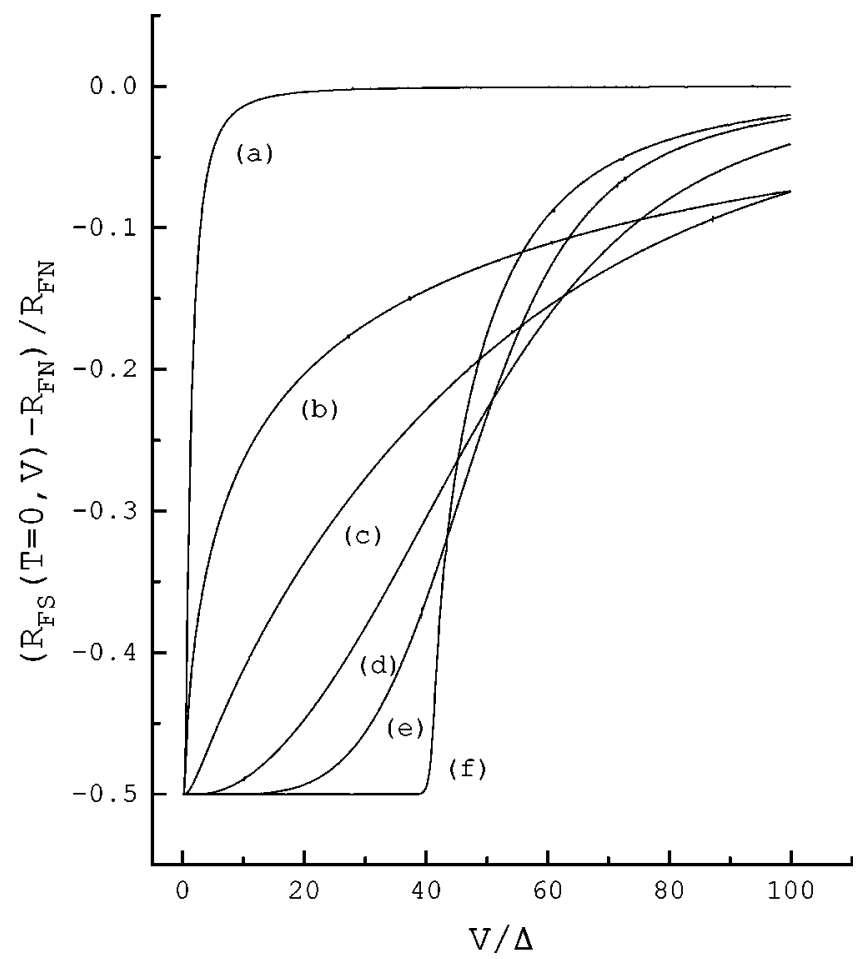

FIG. 9. Resistance change $\left[R_{\mathrm{FS}}(T=0, V)-R_{\mathrm{FN}}\right] / R_{\mathrm{FN}}$ as a function of the bias voltage $V$. The ferromagnetic wire is described by the polarization $\gamma=0$, and the interface resistance is $R_{\mathrm{BN}}$ $=0.05 R_{\mathrm{F}}$. Curve (a) corresponds to no electron-phonon interaction $\kappa=0$. Curves (b), (c), (d), and (e) correspond to an intermediate electron-phonon interaction $\kappa=10^{2}, \kappa=10^{4}, \kappa=10^{6}$, and $\kappa=10^{8}$, respectively. Curve (f) is for the case of a strong electron-phonon interaction $\kappa=\infty$.

will turn out that most of the experimental results can be understood on the basis of our calculations. Both experimental arrangements we will discuss below contain F-S junctions where the superconductor and the ferromagnet overlap in a certain region. The current redistribution in these junctions will play an important role in the following. Let us therefore introduce parameters characterizing these junctions: the resistance of the interface is called $R_{\mathrm{BN}}$ in accordance with our previous consideration. Additionally $R_{\mathrm{SJ}}$ will be the resistance of the superconducting part of the overlap junction in the normal state and $R_{\mathrm{FJ}}$ the resistance of the ferromagnetic part of the overlap junction.

In the experiment by Giroud et al. ${ }^{6}$ a nonmonotonic behavior of the resistance below the superconducting critical temperature was observed. The sample consisted of a ferromagnetic wire, the resistance of which was measured in a four-point arrangement. At some point a superconducting strip was on top of the wire. In a second sample two such strips were present and the resulting resistance change was twice as big as in the case of one strip. Since our formulation is based on a single interface and no coherent coupling between the two superconducting strips was found experimentally, we concentrate here on the sample with one strip. The resistance change in the two-strip sample is then simply twice that for the single-strip sample. The experimental arrangement is such that in the region of the strip the current is redistributed among the ferromagnet and the superconductor. In Appendix A we introduce a simple quasi-one-dimensional 


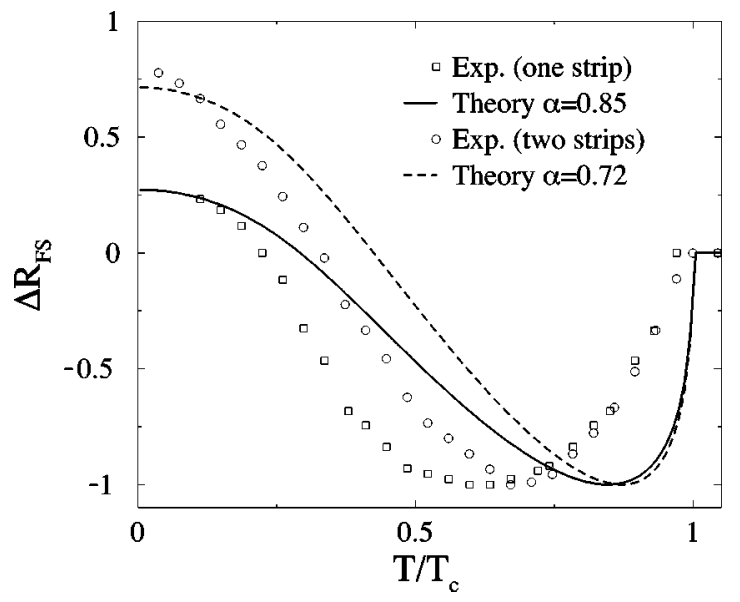

FIG. 10. Comparison of the experiment of Giroud et al. (Ref. 6) and theoretical calculations. The two experimental data sets are for the sample with one superconducting strip (squares) and for the sample with two superconducting strips (circles). The theoretical curves are obtained from Eq. (34) with the following parameters: $R_{\mathrm{F}}=100 R_{\mathrm{BN}}$, spin relaxation length $l_{\mathrm{sf}}=0.012 L$, conductivity polarization $\gamma=0.3$, and a double-interface contact. All curves are normalized to the respective maximal negative value.

model to calculate the effective resistance, these results being used for comparison with experiment. The resistance of the superconducting $\mathrm{Al}$ strip is $0.4 \Omega$, the resistance of the ferromagnetic part below the strip is $10 \Omega$, and the resistance of the interface is estimated to be $0.1 \Omega$. Since the measured resistance change of the $\mathrm{F}$ wire shows no signature of the vanishing of the resistance of the superconducting part, we believe that the real interface resistance is higher than estimated in Ref. 6, in particular higher than $R_{\mathrm{SJ}}$. This yields a total resistance of $R_{\mathrm{eff}}=2\left(R_{\mathrm{FJ}} R_{\mathrm{BN}}\right)^{1 / 2}$, which is approximately of the order of a few $\Omega$. A resistance change of the interface resistance $\delta R_{\mathrm{B}}$ will then lead to a change of the effective resistance $\delta R_{\mathrm{eff}}=\left(R_{\mathrm{FJ}} / R_{\mathrm{BN}}\right)^{1 / 2} \delta R_{\mathrm{B}}$, which in the case $R_{\mathrm{FJ}}>R_{\mathrm{BN}}$ is larger than the resistance change of the interface resistance itself. For the experimental values we have $\left(R_{\mathrm{FJ}} / R_{\mathrm{BN}}\right)^{1 / 2} \approx 10$ and thus a resistance change of $\approx 0.2 \Omega$, as observed in the experiment may result from a change of the interface resistance $R_{\mathrm{BN}} \approx 0.1 \Omega$ by $20 \%$.

The results of Petrashov et al. ${ }^{5}$ are more intriguing, since the magnitude of the measured resistance drop in some of the samples seems to be far too large to be explained without a "long-range" proximity effect in the ferromagnet. We will concentrate here on three of the four samples discussed in Ref. 5. In these samples the transport through a long ferromagnetic wire with one ferromagnetic and one superconducting contact is studied; this is in contrast to the experiments of Ref. 6. The geometry is such that the superconducting contact overlaps the ferromagnetic wire at one end and the current has to pass through a tiny piece of the superconductor. The three samples differ in the interface resistance. Two samples with a low interface resistance show large drops of the resistance of the order of 8 and $16 \Omega$, respectively, below the superconducting critical temperature. The third sample has a higher interface resistance $\left(R_{\mathrm{BN}}=41 \Omega\right)$ and shows a small resistance increase of the order of $1.5 \Omega$.

This agrees qualitatively with the results of our model. Indeed, the bigger resistance of the boundary usually means
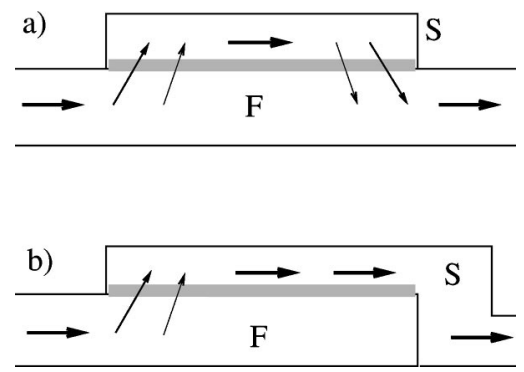

FIG. 11. Schematic picture of current redistribution in an overlap junction. The geometry (a) corresponds to the experiment by Giroud et al. (Ref. 6). If the resistance of the ferromagnet below the overlap region is the highest, a considerable part of the current flows through the interface and the superconductor. The geometry in (b) corresponds to the geometry of Petrashov et al. (Ref. 5). Here the current is forced to leave the contact region through the superconductor.

the formation of a ticker tunnel barrier such that the transmission eigenvalues are shifted towards zero. Our model does predict a resistance decrease for a fairly transparent interface and changes to an increase for a more tunnel-like interface. This is shown, e.g., in Fig. 4. However, quantitatively one would expect that the resistance changes below the superconducting transition temperature are always of the order of the boundary resistance itself.

This is obviously not the case in the experiment with $R_{\mathrm{BN}}=41 \Omega$, where the measured resistance change is about 40 times smaller. The first idea is that the resistance drop of the samples with better interface may possibly be accounted for by combining the effect of the current redistribution and the apparent enhancement of the Andreev reflection discussed in Sec. IV. Again we calculate the effective interface resistance in a quasi-one-dimensional model (see Appendix A). In the limit of a small interface resistance $R_{\text {eff2 }}$ $=\left(R_{\mathrm{FJ}} R_{\mathrm{BN}}\right)^{1 / 2}$. A change of the interface resistance again results in an apparently larger change of the effective resistance $\delta R_{\text {eff } 2}=\left(R_{\mathrm{FJ}} / R_{\mathrm{BN}}\right)^{1 / 2} \delta R_{\mathrm{B}} / 2$. We may speculate that the large resistance drop observed in the experiment by $\mathrm{Pe}$ trashov et al. can possibly be explained by this effect together with the observation made in Sec. IV that a spindependent interface may cause another apparent enhancement of the Andreev reflection.

There may be a more radical explanation for a small relative resistance change. In fact, the morphology of the metalferromagnet interfaces has not been yet sufficiently studied. The actual structure of the interface may be complicated. ${ }^{25}$ To illustrate how this can affect the results let us consider a simplistic model of a double interface. We speculate that a thin layer of magnetic alloy separates the ferromagnet and the superconductor. The boundary scattering then occurs in two stages: at the "inner" interface between the ferromagnet and the alloy and at the "outer" interface between the superconductor and the alloy. Since the proximity effect is quenched in magnets, the resistance of the "inner" interface is not affected by the superconducting transition whereas the resistance of the "outer" interface acquires a change described above. This leads to a smaller relative resistance change.

In Fig. 10 we show a comparison between the experimental results of Giroud et al. and our calculation for a contact 


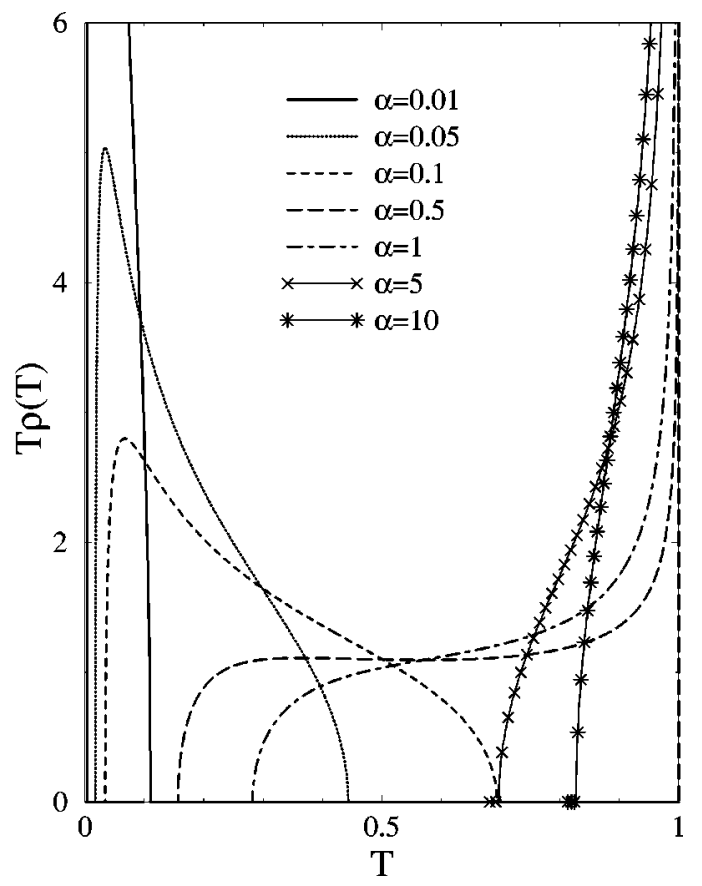

FIG. 12. Transmission eigenvalue distribution for the double interface. The ratio $\alpha=G_{\mathrm{T}} / 2 G_{\mathrm{QPC}}$ is varied between the different curves.

with a transmission eigenvalue distribution of the model just described (see Appendix B). The maximal relative resistance change of the contact is $\approx 16 \%$ in our calculation. According to above considerations this may be enhanced to a measured resistance change of the order of $\approx\left(R_{\mathrm{FJ}} / R_{\mathrm{BN}}\right)^{1 / 2} 16 \% \delta R_{\mathrm{B}}$ $\approx 160 \% \delta R_{\mathrm{B}}$ in agreement with measurements.

\section{CONCLUSIONS}

To summarize, we have calculated the resistance of a diffusive ferromagnetic wire in contact with a superconducting reservoir in the linear and nonlinear regime with purely elastic and inelastic scattering. It has been demonstrated that most of the recent experimental results can be understood in the absence of a superconducting proximity effect in the ferromagnet. Spin accumulation leads to an enhanced resistance below the superconducting transition temperature whereas Andreev reflection can lead to a decreased resistance below the superconducting transition temperature. The competition between these two mechanisms determines the sign of the resistance change. The magnitude of the resistance change is of the order of the interface resistance or the spin-relaxation resistance. Electron heating can dramatically modify the nonlinear response resistance and change the bias dependence by orders of a magnitude.

\section{ACKNOWLEDGMENTS}

A.B. would like to thank B. I. Halperin for discussions. This work was partially supported by the "Stichting voor Fundamenteel Onderzoek der Materie" (FOM) and a Feodor Lynen Fellowship of the "Alexander von HumboldtStiftung" (W.B.) and the Norwegian Research Council (A.B).

\section{APPENDIX A: CURRENT REDISTRIBUTION IN AN OVERLAP JUNCTION}

Here we introduce a quasi-one-dimensional model to account for the redistribution of the current under an overlap junction as used in the experiments. The two geometries we have in mind are depicted in Fig. 11.

To estimate the measured resistance we use the following quasi-one-dimensional model for the current redistribution in the overlap region of length $d$. The currents $I_{F}(x)$ in $\mathrm{F}$ and $I_{S}(x)$ in $\mathrm{S}$ in direction of the ferromagnetic wire follow from Ohm's law

$$
I_{\mathrm{F}}(x)=\frac{d}{R_{\mathrm{FJ}}} \frac{d U_{\mathrm{F}}(x)}{d x}, \quad I_{\mathrm{S}}(x)=\frac{d}{R_{\mathrm{SJ}}} \frac{d U_{\mathrm{S}}(x)}{d x},
$$

where $R_{\mathrm{FJ}(\mathrm{SJ})}$ is the resistance of the ferromagnetic part under (superconducting part above) the contact and $U_{F(S)}$ the respective voltage. Current conservation dictates that

$$
\frac{d I_{\mathrm{F}}(x)}{d x}=-\frac{d I_{\mathrm{S}}(x)}{d x}=\frac{U_{S}(x)-U_{F}(x)}{R_{\mathrm{BN}} d}
$$

is the current per unit length through the contact resistance $R_{B N}$. Boundary conditions are obviously that the total voltage drop is $V$ and no current leaves the system through the boundary to vacuum.

Solving these equations for the geometry of Giroud et al. ${ }^{6}$ we find the effective resistance of this part to be

$$
R_{\mathrm{eff}}=\frac{R_{\mathrm{FJ}}}{R_{\mathrm{FJ}}+R_{\mathrm{SJ}}}\left[R_{\mathrm{SJ}}+\sqrt{\frac{4 R_{\mathrm{BN}}}{R_{\mathrm{SJ}}+R_{\mathrm{FJ}}}} \tanh \left(\sqrt{\frac{R_{\mathrm{FJ}}+R_{\mathrm{SJ}}}{4 R_{\mathrm{BN}}}}\right)\right] .
$$

Of specific interest is the case that $R_{\mathrm{FJ}} \gg R_{\mathrm{BN}} \gg R_{\mathrm{SJ}}$, in which we obtain $R_{\mathrm{eff}} \approx 2\left(R_{\mathrm{FJ}} R_{\mathrm{BN}}\right)^{1 / 2}$.

A calculation similar to the previous for the geometry of Petrashov et al. ${ }^{5}$ leads to an effective measured resistance of

$$
R_{\mathrm{eff} 2}=\frac{\sqrt{R_{\mathrm{FJ}} R_{\mathrm{BN}}}}{\tanh \sqrt{R_{\mathrm{FJ}} / R_{\mathrm{BN}}}}
$$

in the limit of vanishing resistance of the superconductor on top of the ferromagnet. The difference with the previous calculation is that here the current enters the junction through the ferromagnet, but has to leave the junction through the superconductor.

\section{APPENDIX B: TRANSMISSION EIGENVALUES OF A DOUBLE INTERFACE}

The distribution of transmission eigenvalues of a double interface as described in the text can be found with the technique described in Ref. 26. For details we refer to these articles. We model the double interface by a ballistic contact and a tunneling barrier in series. The tunneling barrier of conductance $G_{\mathrm{T}}$ models the sharp drop in the potential due to the band structure mismatch, whereas the region close to that interface is treated as a collection of unit transmission channels with a total conductance $G_{\mathrm{QPC}}$. The distribution of transmissions can be found from the solution of 


$$
I(\Phi)=G_{\mathrm{T}} \sin (\Phi-\theta)=2 G_{\mathrm{QPC}} \tan \left(\frac{\theta}{2}\right) .
$$

The distribution of transmission eigenvalues $\rho(T)$ is found by analytic continuation into the complex plane:

$$
\rho(T)=\frac{1}{e^{2}} \frac{1}{T \sqrt{1-T}} \operatorname{Re}\left[I\left(\pi+2 i \operatorname{acosh} \frac{1}{\sqrt{T}}\right)\right] .
$$

The dependence on the two separate conductances may be eliminated in favor of the total conductance of the contact $G_{\mathrm{BN}}=G_{\mathrm{T}} G_{\mathrm{QPC}} /\left(G_{\mathrm{T}}+G_{\mathrm{QPC}}\right)$ and the ratio of the two $\alpha$
$=G_{\mathrm{T}} / 2 G_{\mathrm{QPC}}$. The transmission eigenvalue distribution then only depends on $\alpha$. It is plotted in Fig. 12 for several values of $\alpha$. For small values of $\alpha$ the contact is dominated by the tunnel barrier, resulting in a shift of the transmission eigenvalues to lower values and a gap above a certain $T$. Higher $\alpha$ 's shift the distribution to larger transmission eigenvalues and a gap opens up for low transmission eigenvalues. For a range $0.1 \lesssim \alpha \lesssim 0.5$ the distribution is restricted to a finite interval of transmission eigenvalues. At even higher values of $\alpha$ the upper gap closes and the distribution becomes more and more peaked at $T=1$.
${ }^{1}$ Articles in Mesoscopic Superconductivity, edited by F. W. J. Hekking, G. Schön, and D. V. Averin, [Physica B 203, 201 (1994)]; articles in Mesoscopic Electron Transport, edited by L. L. Sohn, L. P. Kouwenhoven, and G. Schön (Kluwer, Dordrecht, 1997); C. Bruder, Supercond. Rev. 1, 261 (1996); C.J. Lambert and R. Raimondi, J. Phys.: Condens. Matter 10, 901 (1998); W. Belzig, F. K. Wilhelm, C. Bruder, G. Schön, and A. D. Zaikin, Superlattices Microstruct. 25, 1251 (1999).

${ }^{2}$ P. M. Levy, Solid State Phys. 47, 367 (1994); M. A. M. Gijs and G. E. W. Bauer, Adv. Phys. 46, 285 (1997).

${ }^{3}$ A. Brataas, Yu. V. Nazarov, J. Inoue, and G. E. W. Bauer, Phys. Rev. B 59, 93 (1999); Eur. Phys. J. B 9, 421 (1999); D. Huertas Hernando, Yu. V. Nazarov, A. Brataas, and G. E. W. Bauer, Phys. Rev. B 62, 5700 (2000).

${ }^{4}$ S. K. Upadhyay, A. Palanisami, R. N. Louie, and R. A. Buhrman, Phys. Rev. Lett. 81, 3247 (1998); R. J. Soulen, Jr., J. M. Byers, M. S. Osofsky, B. Nadgorny, T. Ambrose, S. F. Cheng, P. R. Broussard, C. T. Tanaka, J. Nowak, J. S. Moodera, A. Barry, and J. M. D. Coey, Science 282, 85 (1998); S. K. Upadhyay, R. N. Louie, and R. A. Buhrman, Appl. Phys. Lett. 74, 3881 (1999);

${ }^{5}$ V. T. Petrashov, I. A. Sosnin, I. Cox, A. Parsons, and C. Troadec, Phys. Rev. Lett. 83, 3281 (1999); J. Low Temp. Phys. 118, 689 (2000).

${ }^{6}$ M. Giroud, H. Courtois, K. Hasselbach, D. Mailly, and B. Pannetier, Phys. Rev. B 58, R11 872 (1998).

${ }^{7}$ M. D. Lawrence and N. Giordano, J. Phys.: Condens. Matter 8, L563 (1996); 11, 1089 (1999).

${ }^{8}$ M. J. M. de Jong and C. W. J. Beenakker, Phys. Rev. Lett. 74, 1657 (1995); A. Brataas and G. E. W. Bauer, Europhys. Lett. 26, 117 (1994).

${ }^{9}$ V. I. Fal'ko, C. J. Lambert, and A. F. Volkov, Pis'ma Zh. Éksp. Teor. Fiz. 69, 497 (1999) [JETP Lett. 69, 532 (1999)]; F. J. Jedema, B. J. van Wees, B. H. Hoving, A. T. Filip, and T. M. Klapwijk, Phys. Rev. B 60, 16549 (1999).

${ }^{10}$ A. Kadigrobov, R. I. Shekhter, M. Jonson, and Z. G. Ivanov, Phys. Rev. B 60, 14593 (1999); E. A. Demler, G. B. Arnold, and H. R. Beasley, ibid. 55, 15174 (1997).
${ }^{11}$ R. Seviour, C. J. Lambert, and A. F. Volkov, Phys. Rev. B 59, 6031 (1999); M. Leadbeater, C. J. Lambert, K. E. Nagaev, R. Raimondi, and A. F. Volkov, ibid. 59, 6031 (1999).

${ }^{12}$ S. Kashiwaya, Y. Tanaka, N. Yoshida, and M.R. Beasley, Phys. Rev. B 60, 3572 (1999); I. Zuti'c and O. T. Valls, ibid. 60, 6320 (1999); 60, 16322 (1999); 61, 1555 (2000); A. Dimoulas, ibid. 61, 9729 (2000); R. Mélin, Europhys. Lett. 51, 202 (2000).

${ }^{13}$ G. E. Blonder, M. Tinkham, and T. M. Klapwijk, Phys. Rev. B 25, 4515 (1982).

${ }^{14}$ F. Zhou and B. Spivak, cond-mat/9906177 (unpublished).

${ }^{15}$ P. B. Allen, Phys. Rev. Lett. 59, 1460 (1987).

${ }^{16}$ A. V. Zaitsev, Zh. Éksp. Teor. Fiz. 86, 1742 (1984) [Sov. Phys. JETP 59, 1015 (1984)].

${ }^{17}$ Yu. V. Nazarov, Superlattices Microstruct. 25, 1221 (1999).

${ }^{18}$ A. Brataas, Yu. V. Nazarov, and G. E. W. Bauer, Phys. Rev. Lett. 84, 2481 (2000).

${ }^{19}$ K. M. Schep and G. E. W. Bauer, Phys. Rev. Lett. 78, 3015 (1997).

${ }^{20}$ O. N. Dorokhov, Pis'ma Zh. Éksp. Teor. Fiz. 36, 259 (1982) [JETP Lett. 36, 318 (1982)]; A. D. Stone, P. A. Mello, K. A. Muttalib, and J. L. Pichard, in Mesoscopic Phenomena in Solids, edited by B. L. Altshuler, P. A. Lee, and R. A. Webb (NorthHolland, Amsterdam, 1991).

${ }^{21}$ S. N. Artemenko, A. F. Volkov, and A. V. Zaitsev, Solid State Commun. 30, 771 (1979).

${ }^{22}$ A. V. Zaitsev, Pis'ma Zh. Éksp. Teor. Fiz. 51, 35 (1990) [JETP Lett. 51, 41 (1990)].

${ }^{23}$ In the actual calculation we have approximated the temperature dependence of the superconducting gap by $\Delta(T)$ $=1.76 T_{\mathrm{c}} \tanh \left[1.74\left(T_{\mathrm{c}} / T-1\right)^{1 / 2}\right]$.

${ }^{24}$ K. M. Schep, P. J. Kelly, and G. E. W. Bauer, Phys. Rev. Lett. 74, 586 (1995); K. Xia et al. (unpublished).

${ }^{25}$ V. V. Petrashov (private communication).

${ }^{26}$ Yu. V. Nazarov, Phys. Rev. Lett. 73, 134 (1994); see also Yu. V. Nazarov, in Quantum Dynamics of Submicron Structures, edited by H. A. Cerdeira, B. Kramer, and G. Schön (Kluwer, Amsterdam, 1995). 